\title{
Evolución de la Universidad en la Sociedad del Aprendizaje y la Enseñanza. El valor de las competencias en el desarrollo profesional y personal
}

\author{
Emilio Álvarez-Arregui (*) \\ Universidad de Oviedo (España)
}

Profesor Titular de la Universidad de Oviedo en el Departamento de Ciencias de la Educación, actualmente forma parte del Equipo rectoral de esta institución como director del Área del Instituto de Investigación e Innovación Educativa. Ha sido investigador principal en proyectos autonómicos, nacionales e internacionales y actualmente está trabajando en el desarrollo de modelos de desarrollo de competencias con universidades, institutos de investigación, compañías, administraciones, consejerías, agentes sociales y plataformas de aceleración de la innovación de diferentes países americanos y europeos. Tiene más de dos centenares de publicaciones en revistas y libros relacionadas con estas temáticas. Ha realizado estancias docentes e investigadoras en universidades españolas (Sevilla, Huelva, León, Islas Baleares, Jaén, UNED...) y extranjeras (Uruguay, Chile, México, Letonia, Portugal, EEUU, Reino Unido, Irlanda, Brasil, Suiza...). Es miembro de la Sociedad Española de Pedagogía, del Grupo "Universidades y Educación Inclusiva”, del "Servicio Español para la Internacionalización de la Educación (SEPIE)", de la "Red Europea de Educación Inclusiva y Discapacidad incluDed"; del "Fórum Europeo de Administradores de la Educación (FEAE)"; de la Red Transdisciplinar de Investigación Educativa (RETINDE); de la Red de Universidad Inclusivas (RUIE); de la Red de Institutos de Investigación Educativa de España (RIIE); de la Red Euroamericana Alfamed; de la Red Iberoamericana de Evaluación de la Calidad Educativa de la Educación Superior (RIAICES); de la Red Universitaria de Estudios de Posgrado y Educación Permanente (RUEPEP); y de la Red UNESCO en el Principado de Asturias, entre otras. Es director de la Revista Aula Abierta.

ORCID: https://orcid.org/0000-0002-4657-753X alvarezemilio@uniovi.es

\section{RESUMEN}

Sociedad, Educación y Universidad han estado interrelacionadas en base a los condicionantes económicos, sociales, estructurales, tecnológicos, políticos, jurídicos y funcionales concurrentes en cada momento histórico. Las decisiones adoptadas en este ecosistema han sido, son y serán controvertidas porque no son neutras ideológicamente lo que ha acabado determinando culturas organizativas y funcionales ligadas a los sistemas de poder y conocimiento vigentes que se legitiman en las áreas geográficas en las que se integran. En este artículo hacemos un recorrido por el pasado, presente y futuro de la universidad desde diferentes miradas desde las que se avala la necesidad de evolución de estas organizaciones desde un enfoque sistémico y multidisciplinar lo que requiere alinear el aprendizaje, la enseñanza, la formación, la investigación, la gestión, el talento y el compromiso desde un paradigma integrador que nos permita generar las innovaciones necesarias para adaptarnos y proyectarnos en un entorno que no tiene antecedentes pretéritos y del que desconocemos su evolución. En consecuencia, es preciso que la universidad asuma el valor real de las competencias para que las personas concurrentes en estas instituciones aprendan y enseñen a aprender y emprender si quieren adaptarse a un contexto de cambio rápido y complejo.

Palabras Clave: aprendizaje, enseñanza, formación, investigación, competencia e innovación educativa.

\section{Contextualización}

La Universidad, como institución educativa de referencia de la Educación Superior, se ha adaptado a los condicionantes económicos, sociales, estructurales, tecnológicos, políticos, jurídicos y funcionales concurrentes en cada momento histórico. Las decisiones adoptadas han sido, son y serán controvertidas porque no son neutras ideológicamente, porque afectan diferencialmente a las instituciones y porque no discriminan las singularidades de los colectivos participantes. Esta situación ha generado unas culturas organizativas y funcionales ligadas a los sistemas de poder vigentes que se han legitimado social, política, jurídica, académica y científicamente lo que ha tenido consecuencias de diferente pronóstico en los ámbitos y entornos que le son propios.
La Sociedad actual se va interpretando como un ecosistema glocal donde se actúa global y situacionalmente simultáneamente generando y utilizando una economía de datos, información, conocimiento y sabiduría para transitar hacia una Sociedad del Aprendizaje y la Enseñanza Permanente lo que está suponiendo un cambio de hondo calado en los ejes que guían a las personas físicas y jurídicas. La problemática que se está detectando en las decisiones que se están adoptando es que en ellas se vienen primando las decisiones a corto y medio plazo sin valorar suficientemente sus consecuencias a largo plazo. Tampoco parece que se quiera entender que los cambios estructurales no vienen acompañados de cambios culturales lo que está generando desviaciones entre las intenciones de los promotores de la innovación y en los resultados que se obtienen. Ante estas y otras situaciones se 
hace necesario despejar incógnitas en múltiples ecuaciones dinámicas para reconducir los debates emergentes que enfrentan a los administradores y a los receptores de los servicios educativos. A manera de introducción incorporamos algunas cuestiones que están sobre el tablero de juego de las organizaciones universitarias en estos momentos, a saber:

¿están preparados los docentes universitarios para transmitir los principios, valores, actitudes y competencias que se demandan como necesarias desde el entorno político, académico, social y laboral en sus entornos culturales de referencia?

¿en qué medida pueden desarrollar estas instituciones en sus ciclos formativos competencias tales como habilidades sociales, liderazgo, capacidad de trabajo en equipo, gestión del estrés, inteligencia emocional, disciplina, responsabilidad, compromiso, productividad, predisposición, actualización continua, lealtad o pensamiento sistémico?

¿es realista seguir presentando las certificaciones universitarias como fuentes o indicadores de competencias de los usuarios, de estabilidad, selección, estatus en la comunidad y desarrollo social, profesional, cultural, político y personal?

¿es viable y/o necesario diseñar un currículum universal para facilitar un aprendizaje personalizado para todas las personas, garantizar la igualdad de oportunidades, promover el trabajo multidisciplinar o favorecer la colaboración desde la competencia y la estandarización?

¿es posible reorientar el individualismo, el credencialismo y la burocratización hacia la colegialidad, el trabajo en equipo, la autonomía, la flexibilidad, la creatividad o la innovación con el marco legislativo actual?

¿se valora realmente la responsabilidad social desde los ámbitos académicos, sociales, políticos, sindicales y empresariales sobre la gestión, los recursos, la financiación y la selección de personal en la universidad o son los grupos de poder internos y sus intereses los que orientan los procesos?

¿cómo pueden afectar las reacciones de las grandes corporaciones ante el monopolio de certificación de las universidades?

¿la formación dual puede generar puentes más sólidos entre la empresa, la academia, los agentes sociales y el entorno político-jurídico de manera generalizada, debe de plantearse de manera diferencial, debe dársele un enfoque multisectorial...?

¿los sistemas de formación inicial, de acceso y continua dan respuesta a las necesidades profesionales de los agentes educativos universitarios?

¿deberían negociarse con los agentes sociales nuevos tiempos para la formación permanente y la conciliación familiar a nivel global, europeo, estatal, regional e institucional?

¿dónde queda la creatividad, la solución de problemas, el desarrollo de proyectos, el control de las emociones y el impacto del conocimiento en los usuarios finales?

¿qué cambios culturales ha generado la Estrategia 2020 en el ámbito universitario y como se interpreta la Agenda 2030?..

Estas y otras cuestiones que se debaten en distintos foros están sometiendo al ecosistema socioeducativo a múltiples presiones, tal y como avalan los informes internacionales, nacionales e institucionales (CES, 2018) en los que alertan acerca de la perdida de eficacia de los sistemas educativos formales. Esta primera lectura apunta ya que un modelo que resultaba válido en épocas pretéritas es cuestionando de manera reiterada ante el incremento exponencial de las demandas de cambio. Esta situación obliga a las personas físicas y jurídicas a apoyarse en la innovación permanente y en un aprendizaje y enseñanza multidisciplinar continuado en todos los ámbitos como estrategia de adaptación. El problema es que no encuentra un eco claro en las instituciones de Educación Superior (Álvarez-Arregui, 2017, 2018, 2019).
En los siguientes apartados aportamos diferentes miradas interrelacionadas de la universidad con la intención de ayudarnos a entender la evolución de sus misiones y disponer así de un mayor número de referentes para construir una visión sistémica, sistemática y flexible que nos permita proyectarnos hacia un futuro incierto fortaleciendo los puntos de encuentro, negociando las disensiones y avalando los procesos de convergencia y divergencia para abordar los problemas y desplegar proyectos. Este planteamiento requiere alinear el aprendizaje, la enseñanza, la formación, la investigación, la gestión, el talento y el compromiso desde un paradigma integrador donde concurran las culturas política, académica, empresarial y social para generar las innovaciones educativas necesarias desde las que dar respuestas a un entorno que no tiene antecedentes pretéritos y del que desconocemos su evolución. En consecuencia, es preciso que la universidad reflexione y asuma el valor real de las competencias para que las personas aprendan y enseñen a aprender, enseñar y empreder si quieren adaptarse a un contexto de cambio rápido y complejo.

\section{Una mirada retrospectiva de la Universidad: etimología, visio- nes y modelos}

Una primera aproximación de la palabra "universidad" nos retrotrae a su raíz etimológica del latín donde el término "universĭtas, -ātis" hace referencia a la síntesis de unus (uno) y verto (girado), es decir, "girado hacia uno". "Verto" también se refiere a "verter, derramar y arrojar", pero también a "versar" que es dar la vuelta a algo, tratar un asunto; de ahí que alguien versado se haya asociado a alguien ilustrado. Además, si consideramos que verso procede de universo, el todo, la totalidad, lo que a ello rodea (unus, versus) nos encontramos con que Universidad hace referencia a un objeto complejo cuyas partes se relacionan con al menos alguno de los demás componentes (Bunge, 1989: 196).

En su uso original Universidad designó al colectivo docente (universitas magistrorum) y, posteriormente, al alumnado (universitas scholarium) compartiendo raíz con universo y universal. Por tanto, universidad sería el punto donde todo se une y gira, es totalidad de estudios y estudiosos que se mueven y cambian en conjunto (López-Gómez, 2015). Esta unidad se entroncará con comunidad (communitas) por lo que universidad vendría a ser una búsqueda constante en común-unidad y universitas se identificará con una corporación o comunidad orientada hacia una meta común donde el genitivo determinaba la naturaleza del espacio, donde sus miembros tienen intereses comunes por lo que adquieren un estatuto legal propio e independiente y donde se consolidará como primera misión de estas instituciones la transmisión y la producción de conocimiento (Zonta, 2000).

La universidad medieval se nutrirá en sus orígenes de referentes plurales (griego, cristiano, judío y árabe) creando un contexto adecuado para el cultivo de los saberes lo que las identificará como "torres de marfil" herméticas si bien siempre se enfatizará su valor como eje de desarrollo social y comunitario. Como es lógico su desarrollo no será homogéneo e irán emergiendo situacionalmente dos enfoques, uno más horizontal - ampliación de conocimientos- y otro más vertical -profundización en cada campo de conocimiento- lo que derivará a la larga en dos posiciones, una más humanista, asociada a un conocimiento genérico, y otra más especializada, vinculada a un conocimiento científico más específico (López-Gómez, 2015).

Snow (1987) apuntará sobre este argumento la emergencia de "dos culturas" contrapuestas, la científica y la intelectual, lo que favorecerá la modernización de la universidad por la disección de los saberes fruto de la especialización científica; por la reorientación docente y formativa medieval hacia la investigación y la aca- 
demia; por el progresivo alejamiento de la universidad respecto a la tradición cristiana; por la aproximación creciente de la institución al estado; y por el reconocimiento de los principios básicos académicos de libertad de pensamiento y libertad docente.

El Renacimiento, la Ilustración, la Primera Revolución Industrial, la creación del Estado-Nación en el siglo XIX o las directrices de Humboldt en Berlín fueron otras razones de peso que hicieron emerger y consolidar la segunda misión de la Universidad, la Investigación, que se incardinará de manera desigual en los modelos europeos y americanos que se desarrollaron (Álvarez-Arregui, 2010c).

La universidad moderna evoluciona así como un "circo" con varias pistas donde se presentan la investigación- modelo humboldtiano-, la formación de profesionales- modelo napoleónico- y la educación intelectual de una universidad formativamodelo británico- como tres referentes básicos. Estos modelos serán revisados tras la Segunda Guerra Mundial a medida que el ideal humboldtiano pierda vigencia y la universidad elitista sea puesta en entredicho ante la masificación de la enseñanza superior (1960 - 1975) como resultado del desarrollo económico; el incremento del nivel de vida medio, el aumento de la natalidad y la apuesta de los gobiernos por satisfacer la demanda ciudadana (Trow, 2007). En cualquier caso esta expansión se irá revisando a la baja desde 1975 ante el fuerte aumento del gasto público que generaba la creación de nuevas universidades y por la crisis económica.

En las últimas décadas del pasado siglo la cuestión universitaria se irá considerando como un problema de la sociedad en su conjunto desde la teoría pero en la práctica se hablará de una Universidad Tecnológica (OCDE, 1999) que evoluciona constantemente. A este respecto cabe recordar cómo la noción de "triángulo" que explicaba las interconexiones entre Universidad, Gobierno e Industria derivó hacia la noción de "triple hélice" donde cada una de ellas integra funciones de los diferentes ámbitos (Etzkowitz y Leydersdoff, 1997). Este cambio de enfoque ampliará el horizonte de esta institución tal y como quedó reflejado en Informe Universidad 2000, Informe Bricall, cuando se avaló la importancia de establecer puentes de unión con el entorno social y empresarial, hecho que si bien cuestionaba el modelo alemán sería ratificado en el Informe Tunning (González y Wagenaar, 2003: 248). Esta nueva visión no se generará por azar sino como resultado de la concurrencia de varias macrotendencias que se fueron consolidando desde finales del siglo XX de manera interdependiente, nos referimos a la glocalización (Castells, 1999, 2000), el neoliberalismo (Bell, 1991; Gimeno Sacristán, 2001) y al desarrollo científico-tecnológico exponencial (Dosi, Freeman, Richard, Silverberg and Soete, 1990). Las repercusiones del nuevo marco no se han hecho esperar y están haciendo germinar nuevos modelos económicos, políticos, jurídicos, laborales y sociales que obligan a las personas físicas y jurídicas a una adaptación permanente al cambio lo que se ha trasladado necesariamente a unas instituciones universitarias que se ven abocadas a un proceso de reestructuración y reculturización continuado que está teniendo efectos diferenciales en función de las historias, estrategias y capacidad de financiación de estas instituciones_Álvarez-Arregui, 2017).

La tercera misión de la universidad emerge así como consecuencia lógica de un entorno sociolaboral que pretende fortalecer como ejes vertebradores el Emprendimiento, la Innovación y el Compromiso Social (Bricall, 2000; Álvarez-Arregui, 2018). Esta institución irá asumiendo así un enfoque más gerencial al pedirle que sea una organización excelente que tiene que contribuir al desarrollo económico y social de la zona en las que se ubica compitiendo, colaborando y asociándose con empresas, instituciones, asociaciones y centros tecnológicos en los ámbitos autonómicos, nacionales e internacionales, pero también que expandan valores cívicos y humanos (Álvarez-Arregui, 2019).

El aumento de los requerimientos hacia la Universidad se ha traducido en un desgaste que se manifiesta en diferentes tipos de descontento que ha sido mayor o menor en función de las áreas geográficas. En el caso de España, la respuesta no se ha hecho esperar y en la Conferencia de Rectores de las Universidades Españolas (CRUE) del 22 de abril de 2019 se presentó un decálogo de peticiones a los partidos políticos para mejorar la Educación Superior si se quieren abordar estas cuestiones con unas garantías mínimas de éxito. Entre otras demandas se solicitaba en este documento una nueva ley de universidades, financiación sostenible, aumento y estabilización de las plantillas, mejora de la docencia, apoyo a la investigación, transferencia e innovación, defensa de la igualdad de oportunidades y de los derechos de los estudiantes, mejora de la gestión, impulso de la internacionalización, transparencia y rendición de cuentas. En este escenario se han abierto múltiples debates que demandan arbitrajes entre los administradores y los administrados de lo educativo ya que todas las partes tienen que tener una ganancia. Se hace así necesario construir un MAPAN - Mejor Alternativa Posible a un Acuerdo Negociado (Fisher, Ury y Patton, 2002) desde una reflexión crítica glocal y con una hoja de ruta, la Agenda 2030, que se ha presentado en nuestro entorno cultural como la brújula que indica el camino a seguir y el contenido del debate en los próximos años.

\section{Una mirada crítica hacia la situación actual: la estandarización y la cuantificación}

La capacidad de respuesta de las universidades quedará constreñida o favorecida situacionalmente en base a como se articule el marco normativo. Por tanto, los gobiernos tienen una cuota de responsabilidad importante cuando asumen compromisos públicamente, cuando toman decisiones insuficientemente fundamentadas, cuando las iniciativas que se quieren promover no se traducen en la práctica cómo se espera, cuando no se explican las intenciones de manera razonable, cuando se promueven políticas cortoplacistas o cuando los valores que transmiten no se derivan de los principios éticos que dicen defender, entre otras cuestiones.

Atendiendo a este planteamiento Europa ha venido revisando su modelo universitario en clave sociopolítica poniendo más o menos énfasis en la economía, las condiciones laborales, las competencias, los equilibrios sociales o los desarrollos territoriales en diferentes momentos. Los efectos de las decisiones adoptadas han supuesto un rediseño global que ha afectado a todas las universidades en general si bien ha tenido repercusiones diferenciales en los países, las instituciones, los profesionales y los usuarios. Prueba de ello son los puestos que se alcanzan en los rankings de universidades, así en el de Shanghai de 2019 $\mathrm{y}$ anteriores nos encontramos con luces y sombras en base a los resultados alcanzados en nuestro entorno ya que Estados Unidos domina la clasificación mundial sistemáticamente año tras año. A manera de recordatorio cabe indicar que actualmente sitúa a 17 universidades en los 20 primeros puestos, Harvard $\left(1^{a}\right)$, Stanford $\left(2^{\underline{a}}\right)$, MIT $\left(4^{\underline{a}}\right)$ y Berkeley $\left(5^{\underline{a}}\right)$ y a 120 universidades entre las quinientas primeras del mundo. En Europa, el Reino Unido sitúa 34 (8 en las 100 primeras), Alemania 27 (4 en las 100 primeras) y Francia 21 (3 en las 100 primeras) lo que nos informa de una debilidad constatable del EEES en Educación Superior en cuanto a excelencia investigadora mundial en los países que podríamos denominar punteros. 
Estas diferencias pueden interpretarse como lógicas si tenemos en cuenta los niveles de desarrollo y de inversión que hacen los diferentes países por lo que sería esperable que se incrementen las distancias sino cambian las tendencias que se van consolidando. A manera de ejemplo podemos recordar como la contratación en los campus y/o en los centros de investigación norteamericanos se ha acogido a más de 400.000 investigadores formados en Europa en los últimos años. En este escenario tampoco se puede obviar que en el área asiática, China, India y el Medio Oriente se siguen manteniendo y/o incrementando las inversiones en sus instituciones universitarias con lo que crecen las ofertas a los investigadores para ayudar a mejorar esos rankings lo que capitaliza o descapitaliza a las instituciones en cuanto a los recursos humanos de alta cualificación disponibles y se entra en un mercado de gestión del conocimiento. Si focalizamos la atención en nuestro entorno vemos que España sitúa a 13 centros entre las 500 mejores instituciones a saber: Universidad de Barcelona (174), Universidad Autónoma de Barcelona (202),Universidad Complutense de Madrid (209), Universidad de Valencia (291), Autónoma de Madrid (302), Pompeu Fabra (398), Universidad de Granada (366), Politécnica de Valencia (444), Universidad de Oviedo (481), Universidad de Sevilla (485), Universidad de las Islas Baleares y Universidad del País Vasco (401-500), otras 25 universidades españolas se sitúan entre los puestos 500 - 1000.

Aunque son muchas las lecturas que pueden hacerse vamos a referirnos a las que se incorporan desde la clave política, así el gobierno de España indicó que tenemos 47 universidades públicas que imparten grado presencial y de ellas, 38 están entre las 1.000 mejores y solo la Universidad privada de Navarra está entre ellas lo que se justifica como un descuido o desinterés por la investigación y la transferencia de conocimiento desde estas entidades. Esta presentación de los datos es problemática por parcial ya que es susceptible de ser reinterpretada ideológicamente en distintos foros y más cuando se identifica la globalización neoliberal con la Educación Superior o cuando se indica que la situación actual es el resultado de una estrategia prediseñada de la economía del conocimiento dentro del nuevo paradigma de la Gestión Pública donde las políticas de mercado, la obtención de beneficios económicos de lo público o el liderazgo del conocimiento en las universidades queda patente (Saura y Bolivar, 2019). En cualquier caso también hay que ser conscientes que los resultados pueden variarse si se incluyen otros indicadores de medición como la calidad de la formación, la empleabilidad de los egresados o la transferencia real de conocimiento y servicios/ productos que incorporan los usuarios desde la innovación algo que se hace cuando se quieren justificar las políticas y las inversiones (Álvarez-Arregui, 2017, 2019).

A tenor de estas argumentaciones queda patente que las instituciones de investigación superior y las universidades que promueven $\mathrm{I}+\mathrm{D}+\mathrm{i}$ se ven afectadas globalmente por un neoliberalismo estructural que determina distintos estadios de desarrollo institucionales que exigen tratamientos diferenciales en cuanto a las estrategias que promuevan para proyectarse al futuro pero teniendo en cuenta el resto de macrovariables y el estadio de evolución de las instituciones. Aunque ya nos hemos referido a este tema en otras ocasiones (Álvarez-Arregui, 2010, 2017, 2018 y Álvarez-Arregui y Rodríguez-Martín, 2015) hemos realizado una clasificación, podría ser otra, de las instituciones universitarias para respaldar nuestras argumentaciones, así distinguimos entre:

1. Organizaciones de Excelencia y Referencia Mundial (Primer nivel). Aquí se situarían las 100 primeras del mundo con proyectos y estrategias multidisciplinares consolida- das en constante desarrollo con proyección regional, estatal, internacional y global. Cumplen los estándares de calidad desde hace años sin problemas.

2. Organizaciones de Excelencia Internacional (Segundo nivel). Aquí situaríamos a las organizaciones entre los puestos 100 y 500 del ranking. Estas instituciones intentan mantenerse o progresar en este espacio diseñando y desarrollando visiones y estrategias integrales e internacionales con amplia difusión, una cobertura financiera estable y una política de alianzas estratégica. En estos casos las instituciones no cumplen todos los estándares pero tienen una positiva valoración en la mayoría de ellos. La Universidad de Oviedo habría logrado incorporarse al final de este grupo en el año 2019 algo que no conseguía desde 2003.

3. Organizaciones de Referencia Regional con Proyectos Internacionales (Tercer nivel). La horquilla se podría extender hasta el puesto 1000. Estas organizaciones tienen prestigio en sus contextos de referencia y son valoradas internacionalmente en algunos ámbitos. Tienen vocación de desarrollar proyectos multidisciplinares con repercusiones en el ámbito regional, nacional e internacional. Tienen dificultades para cubrir sus propios estándares e invierten en redes estratégicas para intentar mejorar su posición.

4. Organizaciones en busca de su Excelencia (Cuarto nivel). Se incluirían aquí a aquellas organizaciones situadas del puesto 1000 en adelante. Como es lógico es muy amplio el abanico de instituciones que nos podemos encontrar este tramo por lo que debería de ser objeto de una mayor segmentación si bien en este caso se utiliza únicamente con fines explicativos. Aquí nos encontramos universidades jóvenes, o que están en fases iniciales de desarrollo o que tienen dificultades de financiación o que no tienen la estructura necesaria para desarrollarse o que son satélites de otras universidades o que están desarrollando proyectos peculiares independientemente de los rankings.

Aunque esta categorización sería una aproximación a una realidad compleja por multidimensional nos permite recordar a los administradores de lo educativo que obren con cautela cuando hacen comparaciones porque es fácil hacer lecturas sesgadas. Así cuando hacen declaraciones sobre sus instituciones para mejorar en los rankings "se olvidan" de indicar que la emergencia de cientos de universidades en el mundo, caso de China, desplazarán progresivamente a las instituciones europeas ante la mayor capacidad de financiación y contratación de las que son objeto por parte de sus gobiernos.

Con respecto a la titularidad podríamos entender un respaldo a las universidades privadas a condición de que reinviertan en la propia institución o en sus zonas próximas de influencia los beneficios que obtienen o una parte importante de ellos, caso de Harvard o Princenton, pero no avalaremos aquellas fórmulas que distribuyen beneficios entre accionistas ya que cuando el ánimo de lucro es prioritario la calidad de la educación y la equidad empiezan a cuestionarse porque se pone en entredicho el derecho a la educación y la igualdad de acceso, algo que dicen garantizar los estados desde sus constituciones.

Los criterios de mercado tampoco pueden orientar toda la oferta curricular hacia las titulaciones más demandadas u orientar la selección del estudiantado a través de su capacidad económica con unos currículums estandarizados sino que deberán desarrollar políticas a medio y largo plazo bien fundamentadas 
y diseñadas. Tampoco parece viable que la explotación de los resultados derivados del conocimiento se haga independientemente del desarrollo regional por parte de los parques tecnológicos o los viveros de empresas (spin-offs) ya que entonces se corre el riesgo de que se oriente la investigación hacia la rentabilidad a corto plazo, se perderá masa para la reflexión crítica, disminuirá, desaparecerá o se enmascarará la responsabilidad social y el acceso a un currículum universal transversal de la educación superior, al menos en el grado, será cuestionada.

Con relación a los rankings también debemos ser conscientes que los listados focalizan la atención en las 500 o 1.000 universidades de los casi 27.000 centros universitarios que existen en el mundo lo que supone una invisibilización encubierta de instituciones, profesionales y alumnado afectado así como de la variedad de situaciones a las que se enfrentan. Es por ello que los administradores de lo educativo deberían hacer saber a la ciudadanía que los criterios de valoración contienen componentes subjetivos (priorización de indicadores, salarios, tiempo en finalizar los estudios, tasas de graduados, en másteres y doctorado, ratio profesorado/estudiantado, sistemas de selección del profesorado, tasa de empleabilidad...) y componentes cuestionables (medir la calidad de la docente utilizando datos como el número de premios de Nobel obtenidos por sus estudiantes, productividad, indicadores bibliométricos, importancia del idioma, ubicación, acceso...) (Álvarez-Arregui, 2019).

Los gestores de lo educativo tampoco pueden seguir desentendiéndose de los aprendizajes adquiridos sobre el desarrollo organizativo y el cambio cultural. A manera de ejemplo recordaremos a Senge (1990) cuando apuntaba hace tres décadas una realidad que sigue vigente en el entorno universitario. En palabras de este autor el verdadero compromiso con las estrategias que se promueven en las organizaciones no es habitual de ahí que sus promotores desarrollen un liderazgo transaccional donde se "vende" una visión a corto plazo para obtener apoyos puntuales pero no conseguirán un respaldo real y sostenible ya que para ello es más apropiado un estilo de liderazgo transformacional distribuido (Álvarez-Arregui, 2002).

En las instituciones universitarias también nos encontramos a colectivos que asumen un alistamiento acrítico que respalda la visión pero que acaba incorporándose de manera rutinaria en los procesos porque no se sienten copartícipes de la misma. Senge (1990) también nos indicaba a este respecto la necesidad de diferenciar entre el acatamiento genuino y el compromiso ya que el primero va más allá en cuanto a las energías y el esfuerzo que se está dispuesto a comprometer un grupo o colectivo para alcanzar la visión. Ante la desobediencia y la apatía este autor nos aconsejaba centrarnos en escuchar e ilusionar desde una óptica realista lo que se traduciría en un liderazgo situacional fruto de una reflexión profunda sobre las razones aducidas. En cualquier caso hay que ser conscientes de que lo mejor es hablar con claridad,evitar la hipocresía y no generar falsas expectativas para que no emerjan enfrentamientos, chantajes encubiertos o conflictos abiertos. Ante posiciones radicales excluyentes poco se puede hacer y será la normativa la que marque la decisión a tomar.

Las caras que se van mostrando nos informan que el camino a seguir por las instituciones de educación superior no será fácil pero será aún más tortuoso si se les sigue exigiendo que se incorporen en sistemas en los que tienen que sistemas escalables "ficticios" bajo el aval de gobiernos y las empresas que están más preocupados por el mantenimiento del sistema vigente que de las necesidades que se cubren a la ciudadanía. Ante esta situación se hace necesario recordar algunos "olvidos" que destacamos a continuación:
- la carrera es desigual porque no todas las instituciones podrán integrarse en los niveles más altos de excelencia porque el punto de partida no es el mismo, hay diferentes recorridos y se pueden cambiar de manera inesperada,

- los estadios de desarrollo institucional no son equivalentes porque las historias de las universidades han generado culturas organizativas singulares que las condicionan,

- la competencia generalizada seguirá favoreciendo el individualismo, la ansiedad y el estrés entre los agentes implicados,

- los datos, la información y el conocimiento empiezan a ser inabarcables ante el desarrollo de la comunicación y la multiplicación de los centros de producción, de distribución y control lo que dificultará la selección de lo relevante sobre lo accesorio en múltiples planos,

- los equipos docentes e investigadores pueden diseñar, innovar, aplicar o experimentar su grado de excelencia o de fracaso independientemente del resto de la institución lo que deriva en una desaceleración continuada de la colaboración, de la formación y del desarrollo sostenible,

- la capacidad de financiación es fundamental por lo que la base presupuestaria y su sostenibilidad tienen impactos diferenciales de distinto pronóstico si se quiere atajar la fuerte endogamia del sistema, el envejecimiento de las plantillas, la precariedad laboral de algunos colectivos, la falta de recursos para retener talento, o la necesidad urgente de armonizar las políticas del Estado con las de las Comunidades Autónomas en Educación Superior en el caso de España y

- las paradojas, las contradicciones e inequidades que han emergido son de difícil pronóstico porque al pasar a formar parte de las culturas organizativas podrían llegar a quebrar los ideales de un EEES que si bien ha seguido las indicaciones del Proyecto Tunning para traducir el conocimiento disponible en conocimiento viable desde la óptica económica y mercantil tiene muchas deficiencias en el desarrollo profesional, institucional y ciudadano.

\section{Una mirada esperanzada}

La dinámica que se ha establecido en las relaciones entre Sociedad, Investigación, Formación e Innovación son un reto para las instituciones universitarias del futuro. Estas organizaciones podrán adaptarse a los nuevos desafíos cuando comprenden sus propias historias, cuando tengan en cuenta sus contextos, cuando den voz a los participantes y cuando desarrollen visiones, misiones, estrategias y acciones que conjuguen la reestructuración y la reculturización de manera simultánea tomando como eje aglutinador los referentes de la Agenda 2030.

Las instituciones universitarias tienen que ser conscientes que la revolución digital ha cambiado las reglas de juego. La acumulación de datos, información y conocimiento es de tal magnitud que obliga a modificar una y otra vez las unidades de medida para poder seguir el ritmo de producción, así se ha pasado del Kilobyte (KB) al Megayte (MB), al Gigabyte (GB), al Terabyte (TB), al Petabyte (PB), al Exabyte (EB), al Zettabyte (ZB) y al Yotattabyte (YB). Esta situación conlleva desarrollar nuevos sistemas de gestión de los datos, la información y el conocimiento para desarrollar la docencia, la investigación y la transferencia lo que ha revitalizado el Big-Data o más recientemente el Blokchain pero no ha tenido en cuenta el cambio cultural y formativo que este hecho lleva aparejado. Otros problemas añadidos a esta hiperproducción de datos, información y conocimiento es que 
provienen de múltiples campos, son volátiles y con alta fragilidad lo que demanda revisar y rediseñar la naturaleza de los procesos de aprendizaje - enseñanza en términos diferentes a las sociedades pretéritas.

La universidad del siglo XXI se ve abocada a reorientar su foco de atención desde la transmisión hacia la generación de contextos de aprendizaje y enseñanza por lo que deberá favorecer y fomentar el desarrollo de nuevas competencias para todas las personas físicas y jurídicas. En el caso de las instituciones universitarias deben explorarse de manera rigurosa nuevas formas de aprendizaje, de enseñanza, de selección de contenidos, de implementación, de aplicación y de organización que genere un conocimiento significativo para el planeta, para los espacios profesionales, para los entornos vitales y para nosotros mismos (Álvarez-Arregui et all. 2017). A este respecto coincidimos con Edgar Morín (1998: 24) cuando señala como función básica de la universidad la de propiciar la reforma del pensamiento para lograr que las personas sean capaces de crear, relacionar, contextualizar y globalizar, en suma, que dispongan de un pensamiento complejo.

En definitiva, la universidad debe preparar ciudadanos activos que sean buenos profesionales (especializados) pero también debe formar a personas polímatas (polifacéticas, pluridisciplinares) dotadas de una mente que les permita reflexionar y analizar de manera sistémica el mundo que les rodea trabajando en equipos pluridisciplinares para adaptarse y dar respuestas a los problemas que emergen en el entorno social, económico, político, laboral, cultural, del ocio y personal.

En este contexto cabe retomar algunos hitos normativos que han sido referentes hasta el momento actual, el primero es el Tratado de Maastricht (1992) donde se establecieron las bases políticas para la educación superior en una nueva Europa, el segundo fue el Programa PISA que actualizó al anterior a través de los registros periódicos de los perfiles académicos y profesionales y el tercero será el Proyecto Tuning que abrió el un nuevo debate educativo con la intención de identificar e intercambiar información y fomentar la colaboración en el espacio europeo para el desarrollo de la calidad, la efectividad y la transparencia (González y Wagenaar, 2006), por último, la Declaración de la Sorbona (1998) promovió que los gobiernos se alineasen para armonizar la educación superior europea. Las recomendaciones europeas que se desprendieron de estos hitos no se han llevado a la práctica como se esperaba porque los países y las culturas organizativas han tamizado las prescripciones lo que nos lleva una y otra vez a situaciones paradójicas y contradictorias. Es lógico, por tanto, que en el escenario actual se está produciendo lo que Lawn y Grek (2012) denominan "chaotic uniformity" donde la educación superior europea navega sin un rumbo claramente definido al tratar de preservar en la práctica la diversidad de las culturas universitarias europeas.

La Agenda 2020 ha intentado en el última década abordar estas situaciones problemáticas que se han ido generando poniendo el foco de atención en la generación de una Plataforma de "Estrategias de Especialización Inteligente" (S3 de la Comisión Europea) planteando abiertamente la importancia de las políticas regionales para el crecimiento inteligente. Para ello se viene prestando asistencia a las autoridades de los estados miembros y a las regiones para optimizar el impacto de la financiación regional desde el denominado triángulo del conocimiento: Educación, Investigación e Innovación [COM(2010)546]. En este proceso, aunque se ha intentado movilizar a las universidades en apoyo al desarrollo regional desde un planteamiento transformacional, los resultados que se están obteniendo presentan luces y sombras por lo que se ha proyectado una nueva visión desde la Agenda 2030 para revitalizar una vez más el papel de las univer- sidades asignándoles un papel clave en todas las fases de las que depende su cumplimiento en los planos internacional, nacional y local. Así se propone para la próxima década que la Universidad genere, transfiera y difunda un conocimiento abierto al servicio de la sociedad, del bienestar y de la sostenibilidad ya que se presupone que cuenta con las capacidades necesarias para realizar una contribución crítica y constructiva al desarrollo sostenible desde sus valores y saberes. Por ello, estas instituciones deberán poner en valor su capacidad de influencia al servicio de un desarrollo global sostenible no sólo respecto al ODS 4 (ahora un cuadrilátero) sino a los 17 objetivos planteados donde destacan la Investigación inter y transdisciplinar (innovaciones y soluciones, implementación local y nacional y creación de capacidades en este ámbito); la Educación (para el desarrollo sostenible, empleos para implementar los ODS, creación de capacidades y movilizar e implicar a la juventud); la Gestión y la Gobernanza (alienadas con los ODS) y el Liderazgo Social (compromiso público, acción, diálogo intersectorial, desarrollo de políticas y abogacía hacia los ODS, movilización y posicionamientos del sector hacia los ODS y compromiso del sector universitario).

El modelo "oficial" adopta una posición emprendedora al intentar conjugar de manera eficiente los intereses e iniciativas de los sectores Público, Privado Mercantil y Tercer Sector postulando a las Fundaciones como posibles polos de atracción de talento. Se aconsejan organigramas más heterogéneos, juntas de gobierno que incorporen miembros externos elegidos / representados en los órganos colegiados (Senado o Claustro Académico) y/o en el Ministerio correspondiente. La Junta de Gobierno designaría y destituiría a los rectores pero también contribuiría a determinar la misión, la visión y la estrategia. También se ve necesario el reforzamiento de la gestión institucional, vinculando la financiación con el desarrollo, el rendimiento y el impacto. La disminución de la regulación tendría como correlato un incremento del control, de la evaluación y de la rendición de cuentas a la autoridad competente. Por otro lado se apunta la necesidad de fortalecer los órganos ejecutivos y de coordinación por lo que el rector podría nombrar no solo a su equipo de gestión sino que también designaría a los decanos y éstos a las direcciones de los departamentos y centros de investigación lo que plantea una estructura de arriba-abajo que conlleva una profesionalización de la gestión y avala una formación continua. En el caso de los equipos de dirección conlleva capacitación en gestión institucional, liderazgo y responsabilidad social desde el momento que se va a trabajar con recursos públicos y privados procedentes de donaciones, mecenazgo, asociaciones con empresas, creación de spin offs o a través de los servicios diferenciales que se determinen.

Estas propuestas son objeto de múltiples debates y matizaciones por lo que no serán fáciles de implementar dado que conllevan, implícitamente, un cambio radical en la forma de entender las instituciones universitarias tal y como las conocemos. Esta situación hace necesario abordar cada caso atendiendo al sentido y valor del conocimiento que genera, a los efectos perversos de la desconsideración de las diversidades culturales, a las diferentes posibilidades de acceso a la financiación, a los efectos de la participación del entorno empresarial en la construcción del curriculum, caso de la formación dual, a la precariedad de muchos contratos laborales, a una creciente estandarización de los curriculums que dificulta la inclusión y el aprendizaje personalizado o al incremento del individualismo por exceso de competitividad.

Antes de cerrar este apartado nos parece necesario recordar a aquellos empleadores que cuestionan la cualificación de los egresados universitarios en iniciativa, responsabilidad, disponibilidad, capacidad de adaptación al cambio, movilidad geográfica o desenvolvimiento en situaciones sociales,... Es decir este 
colectivo pone especial énfasis en la dimensión moral del trabajo porque detectan que las actitudes de los egresados universitarios no muestran un compromiso o capacidad de sacrificio cuando acceden al entorno laboral. Este colectivo entra de lleno en otro punto neurálgico que tendrá que ser objeto de debate en los próximos meses la enseñanza de valores, el saber ser, algo que se ha ido desplazando en los procesos de enseñanza-aprendizaje en las últimas décadas (Álvarez-Arregui, 2018).

Atendiendo a estas iniciativas, a las argumentaciones presentadas y en base a nuestra experiencia cabría indicar que, en nuestra opinión, la mejora del sistema universitario ya no pasa por asociar el desarrollo de las instituciones de enseñanza superior a las variables macroeconómicas o pensar, cuando hay dificultades, que estamos en la parte baja o intermedia de un ciclo económico, sino que tenemos que decidir hacia donde queremos dirigir los recursos disponibles de manera sostenible para abordar y superar una crisis que es estructural, funcional y cultural. Este planteamiento exige realizar un profundo proceso de revisión sobre las competencias que se deben generar desde la universidad pero para ello se requiere una participación real de los ámbitos académicos, político-sindicales, empresariales y sociales porque todos ellos inciden en lo educativo y la responsabilidad social es compartida.

\section{La competencia como referente en una Sociedad del Aprendizaje y la Innovación}

La sociedad siempre ha valorado la cualificación de sus miembros de un modo $\mathrm{u}$ otro por lo que tradicionalmente el trabajo que proporcionaba el sustento de las personas se conseguía a través de competencias que se adquirían fuera de la enseñanza formal. Como es lógico esta situación fue evolucionando hasta considerar aptas a las personas para realizar trabajos cuando sus conocimientos y conductas singulares les permitían realizar una determinada función en organizaciones sociales, laborales y educativas o por cuenta propia.

Esta forma de entender la competencia que se ha ido manteniendo en el tiempo se viene cuestionando en las últimas décadas a medida que se va haciendo cada vez más visible que en la sociedad venidera los conocimientos serán necesarios pero no suficientes para poder desempeñar las funciones en el entorno personal, social y laboral. También se requerirá alta adaptabilidad para adaptarse a un entorno con un alto dinamismo lo que avala en mayor medida que en otras épocas el conocimiento sistémico, el trabajo colaborativo pluridisciplinar, la formación permanente y la innovación.

El problema que este proceso de cambio genera en los países desarrollados queda patente cuando las exigencias a los trabajadores se incrementan, los salarios se estabilizan, los trabajos para toda la vida se cuestionan, aparecen trabajos atípicos como efectos del desarrollo científico tecnológico o se deslocalizan los centros de producción, entre otras cuestiones. Como es lógico el desgaste profesional, el síndrome del quemado, el incremento de la desmotivación, el síndrome del oxidado, la marginación, la explotación y la exclusión encuentran aquí su caldo de cultivo. El crecimiento exponencial de la robotización y la digitalización es otro fiel reflejo de la emergencia de una inteligencia artificial que hace necesario impulsar en todos los niveles nuevas formas de aprendizaje y enseñanza que combinen, retroalimenten e integren las competencias básicas, genéricas, digitales y personales para saber, saber hacer y saber ser un ciudadano cosmopolita.

Las políticas intentan equilibrar los desajustes emergentes pero para ser efectivas deben dotarse de una visión ecosistémica de futuro a corto, medio y largo plazo si quieren anticipar el futu- ro preparando a la ciudadanía. Las acciones por seguir pasan por rediseñar los modelos vigentes a partir de diagnósticos situacionales atentos a los cambios sociales, laborales, culturales, de ocio y personales. A partir de ahí se podrán desarrollar iniciativas que mejoren las competencias y cualificaciones de las personas físicas y jurídicas pero también para fortalecer e incrementar las sinergias entre los ámbitos académicos, sindicales, empresariales y sociales cuando trabajan en proyectos compartidos.

El EEES ya resaltó en sus principios la importancia de la formación por competencias tal y como se formalizó en diferentes normas en los diferentes estados. En España tuvo su reflejo en el RD 1393/2007 donde se indicó que "los planes de estudios conducentes a la obtención de un título deberán (...) tener en el centro de sus objetivos la adquisición de competencias por parte de los estudiantes, ampliando, sin excluir, el tradicional enfoque basado en contenidos y horas lectivas. Se debe hacer énfasis en los métodos de aprendizaje de dichas competencias, así como en los procedimientos para evaluar su adquisición”. La puesta en práctica de este planteamiento no se ha generalizado porque las competencias son "estructuras complejas de procesos que las personas ponen en acción-actuación-creación para resolver problemas y realizar actividades de la vida cotidiana y del contexto laboral-profesional, orientadas a la construcción y transformación de la realidad" (Ruiz Corbella, 2006: 100). Además, en este sistema de formación que se avalaba no sólo a una dimensión meramente formativa (conceptos y procedimientos) sino que adoptaba una dimensión más educativa al incorporar cuestiones axiológicas y actitudinales junto a los contenidos. A este respecto, aunque compartimos la idea de que las competencias deban incorporar los bienes intelectuales intangibles que la tradición universitaria más genuina siempre ha cultivado consideramos un grave error el haber ido reduciendo su potencial en aquellas instituciones que van desviando el valor de lo universitario hacia la medición, la cuantificación y la bibliometrización. Esta perspectiva está olvidando que el proceso de aprendizaje-enseñanza de las competencias requiere un cambio cultural profesional y personal que necesita recursos y esfuerzos sostenidos en el tiempo.

El enfoque from teaching to learning (Barr y Tagg, 1995) o learner centred teaching también nos ha dado a entender que el estudiante construiría su conocimiento a través de la recopilación y síntesis de la información en una cultura colaborativa y compartida. Además, desde la creatividad se generarían las mejores preguntas para desarrollar proyectos desde los que indagar y se entendía el error como aprendizaje con la intención de seguir probando y no tener miedo al fracaso, al final vamos comprobando que ni la primera ni la segunda intención se han generalizado pero lo que sí se ha instalado es una mayor estandarización y burocratización. En este punto cabe puntualizar que ni los gestores, ni los docentes ni los estudiantes han tenido los apoyos deseables para dotarse de las herramientas necesarias para ofrecer, facilitar y desarrollar las competencias que aparecen reflejadas en los programas curriculares que se han desarrollado para adaptarse al EEES. Así, el modelo centrado en el aprendizaje no se ha implementado en la práctica como era deseable de ahí que tendremos que seguir trabajando en esta dirección avalando todas aquellas iniciativas que entiendan que la función docente tiene un alto grado de vinculación con el proceso de orientación y de apoyo de los aprendices en su recorrido académico y profesional a la entrada, durante y a la salida del sistema en el que se haya incorporado (Álvarez-Arregui, 2017).

La tutoría universitaria recobraba ahora todo su protagonismo al establecerse espacios y tiempos específicos donde se podrían establecer procesos de aprendizaje y enseñanza que fuesen 
más allá de la mera resolución de las dificultades derivadas del programa de una asignatura pero al igual que en los casos anteriores no se consideró suficientemente el cambio profesional y cultural que conllevaba por lo que al final se siguen recogiendo en el papel buenas intenciones que no se traducen en la práctica correctamente. Por tanto, cabe hablar de un déficit más, no superado, que tendremos que abordar a través de campañas de sensibilización que vayan acompañadas de una formación continua transversal si se quiere hacer realidad la demanda de participación activa de las personas, entendiéndolas como aprendices en su conjunto.

El concepto de crédito aunque incorporó modificaciones estructurales no siempre han sido funcionales porque al medir el proceso de aprendizaje en horas de docencia y de esfuerzo del aprendiz lo que se hizo fue incrementar artificialmente la complejidad de la planificación, la metodología y la evaluación universitaria pero este hecho no fue acompañado de la suficiente inversión en espacios, tiempos, reconocimiento o formación por lo que cabe afirmar que su impacto ha sido limitado.

El problema una vez más ha sido la dificultad de integrar en el imaginario profesional las nuevas funciones y a diferencia de lo planteado inicialmente lo que se ha fortalecido ha sido el credencialismo, la estandarización y la burocratización. Por tanto, se hace necesario obrar con cautela cuando se habla de competencias observables o demostrables y diferenciar entre un currículum dirigido a la formación para el empleo o un currículum para una formación profesionalizante ya que su sentido cambia en su segunda acepción al hacerse necesario integrar competencias y no generar agregaciones. En este sentido estamos de acuerdo con Le Boterf (2001) cuando señala que no se puede trabajar con una definición débil de la competencia, es decir, no puede entenderse como una suma de conocimientos de saber hacer o de saber estar o como la aplicación de conocimientos teóricos o prácticos. En la mayor parte de los casos se observa una asimilación de la competencia a un hacer fragmentario (...) La lógica de la descomposición mata la competencia. En nuestro caso nos parece más oportuno definir las competencias en términos de conocimiento combinatorio que aglutina todo un conjunto de conocimientos, procedimientos, actitudes y capacidades que se complementan entre sí. De esta manera la persona para actuar con eficacia frente a las situaciones profesionales debe "saber", "saber hacer", "saber estar y ser", "saber aprender" y "hacer saber" incorporando una visión de conjunto de los "tipos" de competencia (Álvarez-Arregui, 2017). A manera de recordatorio presentamos en la tabla adjunta un breve esquema orientativo.

Tabla 1

Competencias técnicas y sociales (Libro Blanco del Título de Grado en Pedagogía y Educación Social, 2004: 155)

\begin{tabular}{|c|c|c|}
\hline \multirow{2}{*}{$\begin{array}{l}\text { COMPETENCIAS } \\
\text { TÉCNICAS }\end{array}$} & \multirow{2}{*}{$\begin{array}{l}\text { Implican el dominio de los } \\
\text { conocimientos y destrezas } \\
\text { específicos de un determi- } \\
\text { nado campo profesional }\end{array}$} & SABER: Conocimientos generales o específicos, teóricos, especulativos o científico técnicos. \\
\hline & & $\begin{array}{l}\text { SABER HACER: Dominio de los métodos y técnicas específicas de determinados campos } \\
\text { profesionales. }\end{array}$ \\
\hline \multirow{3}{*}{$\begin{array}{l}\text { COMPETENCIAS } \\
\text { SOCIALES }\end{array}$} & \multirow{3}{*}{$\begin{array}{l}\text { Incluye motivaciones, va- } \\
\text { lores, capacidad de rela- } \\
\text { ción en un contexto social } \\
\text { organizativo }\end{array}$} & $\begin{array}{l}\text { SABER APRENDER: Capacidad de formación permanente al ritmo de la evolución que se } \\
\text { produce en las profesiones, en la tecnología y en las organizaciones. }\end{array}$ \\
\hline & & SABER ESTAR: Actitudes de comportamiento en el trabajo y formas de actuar e interactuar. \\
\hline & & $\begin{array}{l}\text { HACER SABER: Posibilitar que en el lugar de trabajo se desarrollen aprendizajes, como } \\
\text { resultado de la intercomunicación personal. }\end{array}$ \\
\hline
\end{tabular}

Una aproximación funcional a las competencias que compartimos es aquella que las identifica con la capacidad de movilizar diferentes recursos cognitivos para hacer frente a una tarea en un contexto determinado (Goñi, 2005: 87) porque asocia la capacidad con "algo" que se posee en potencia y se manifiesta en acto cuando se necesita lo que permite diseñar y orientar los procesos formativos hacia la consecución de dicha competencia. Hecha esta aclaración cabe distinguir como componentes de la competencia (ob. cit.):

Una operación mental (Ser capaz de...) - acción mental que tiene que ver con la personalidad caso de los motivos, los rasgos y el autoconcepto -, un objeto (habitualmente llamado conocimiento), un contenido (concreta el objeto -conflictos, organización de espacios, etc.-) y un fin (contexto de aplicación). La fórmula bajo la que se presentaría sería la siguiente:

\section{COMPETENCIA= (Operación Mental + Objeto $)$ + CONTENIDO + FINALIDAD}

Una posible redacción de una competencia sería la siguiente: Persona capaz de diagnosticar, atentamente, una situación de conflicto laboral en el entorno próximo (municipal, comarcal o institucional) y valorar diversas alternativas para posicionarse de manera razonada y responsable por alguna de ellas. En esta definición aparecen operaciones mentales explícitas (analizar, valorar alternativas y decidir) y objetos sobre los que se actúa mentalmente (conceptos y procedimientos necesarios para analizar un conflicto, las alternativas a considerar y los elementos que entran en juego para tomar una decisión, sin dejar de lado las actitudes a movilizar). La acción planteada tiene una finalidad concreta orientada a tomar una decisión responsable si bien es necesario hacer más clarificaciones que ayuden a entenderlo.

La operación es importante porque se entiende una acción interiorizada que se realiza de manera simbólica lo que permite diferenciar las operaciones de las acciones ya que las primeras suponen manipulaciones de símbolos o imágenes metales y las segundas implican objetos del mundo natural (ob. cit.). Por tanto operar es más amplio que actuar y se adecúa mejor al término competencia.

También cabe indicar que toda acción necesita un objeto (en este caso la mente humana es la que manipula) que puede ser:

- Conceptual (sustantivos, formas sustantivadas). Palabras o términos de carácter lingüístico - trabajo, potencia, energía cinética, etc.- que están ligados al saber.

- Procedimental (verbos). Operación/es sobre un objeto. A la expresión escrita de un procedimiento se le denomina protocolo y se asocia con el saber hacer.

- Actitudinal (adverbios y adjetivos). Toda relación humana esta mediada por valores, comportamientos y actitudes 
sobre los que no se opera como con los conceptos o los procedimientos sino como reguladores de las operaciones, es decir se opera con ella, nos referimos al saber ser y estar.

De Miguel (2006: 32-33) abordó esta temática profundizando en los componentes de la competencia. Aquí enfatizó en:

Intención de la persona donde tiene en cuenta:

- rasgos de personalidad, entendidos como aquellas características que se manifiestan físicamente y conllevan respuestas consistentes a situaciones o reacciones (lograr algo único, de difícil consecución) que pueden ser objeto de diferentes grados de intensidad, lo que denominábamos regulación

- motivación se asociará con aquello que la persona piensa o quiere como causa de determinada acción (ir a clase, participar, realizar una práctica, preparar un examen) y lo hace para lograr metas (aprobar una materia, dominar una habilidad, satisfacer una necesidad personal- pertenencia a un grupo-)

- autoconcepto que reflejará actitudes, valores o la propia imagen personal - predisposición al liderazgo, valor concedido a las calificaciones o inclinación a la colaboración-, $\mathrm{y}$, por último,

- conocimiento lo vinculará a la información de la que dispone una persona sobre áreas de contenido que pueden referirse a conceptos, hechos y procedimientos.

Acción - comportamiento

- Destreza o capacidad de la persona para desarrollar una cierta actividad física o mental (operar, relacionarse, diseñar procesos, asumir un riesgo calculado, usar el feedback del profesorado, responsabilizarse, establecer de metas personales, etc.).

Resultados - desempeño personal y/o profesional.

- En una competencia de motivación de logro el resultado se asocia con la mejora continua en cuanto a las horas de estudio /trabajo dedicadas, lecturas y actividades complementarias realizadas, publicación de trabajos o reconocimientos.

- En el caso de innovación cabría relacionarlo con ideas creativas, solución de problemas, posicionamientos críticos novedosos ante determinadas situaciones o modos de estudiar singulares.

En ambos casos las competencias vinculadas a las características de la personalidad (motivación, rasgos, autoconcepto) son menos visibles mientras que el conocimiento y el comportamiento son más identificables. La fórmula incorpora modificaciones a la anterior, a saber:

\section{COMPETENCIA= INTENCIÓN (personalidad + motivación + autoconcepto + conocimiento) + ACCIÓN + RESULTADO}

Algunos autores que han sintetizado las definiciones sobre competencias (Pérez Escoda y Sabariego Puig, 2015) consideran como más relevantes las siguientes características, a saber:

- El concepto es aplicable a las personas de manera individual o grupal.

- Implica tener saber, habilidad para saber hacer y actitudes y conducta para querer hacer o saber estar, los tres interrelacionados entre sí.
- Circunscribe capacidades formales e informales.

- Su comprensión requiere aunar desarrollo, aprendizaje continuo y experiencia.

- Su potencial se vincula a la capacidad para ponerse en acción.

- Incorpora referentes de eficacia contextuales y requiere esfuerzos para transferirse.

En este contexto compartimos con Gonzci y Athanasou (1996) que la educación y la capacitación basada en competencias constituye un marco coherente para el aprendizaje y el desarrollo de una habilidad. En cualquier caso, lo importante es desarrollarlas de forma apropiada o, de lo contrario, no sólo no se potenciarán esas competencias, sino que además se perjudicará la estructura de desarrollo de otras habilidades (Pavié, 2009).

A partir de estas argumentaciones cabe indicar que las competencias han supuesto un cambio de enfoque en la forma de entender el aprendizaje, la enseñanza y la formación en todos los ámbitos y para todas las personas ya que su orientación será determinante en el grado de exclusión e inclusión de las personas en el futuro. Para atajar estas problemáticas muchos países están optando por aumentar la proporción de graduados en STEM (Ciencias, Tecnología, Ingeniería y Matemáticas) dadas las altas tasas de empleo que se asocian a estos ámbitos por su fuerte componente tecnológico pero están detectando que se encuentran más expuestas a una competencia global que ya no se lidera desde Europa porque algunos países asiáticos ya se sitúan en la parte superior a sus graduados en estas titulaciones. La promoción de políticas de fomento de las mujeres en este contexto es otra alternativa al igual que la promoción de estos colectivos en los empleos verdes relacionados con la ciencia y la tecnología.

En este contexto el talento digital es altamente valorado pero también emergen otros procesos sociales que generan demandas laborales inducidas donde el componente humano cobra un nuevo protagonismo en forma de servicios presenciales, nos referimos a los efectos del envejecimiento diferencial de la población en el planeta o la incorporación de las mujeres al mercado de trabajo. Estos hechos han generado nuevas demandas de atención y cuidados a otros colectivos de la población, caso de la atención infantil, de las personas mayores o de las dependientes.

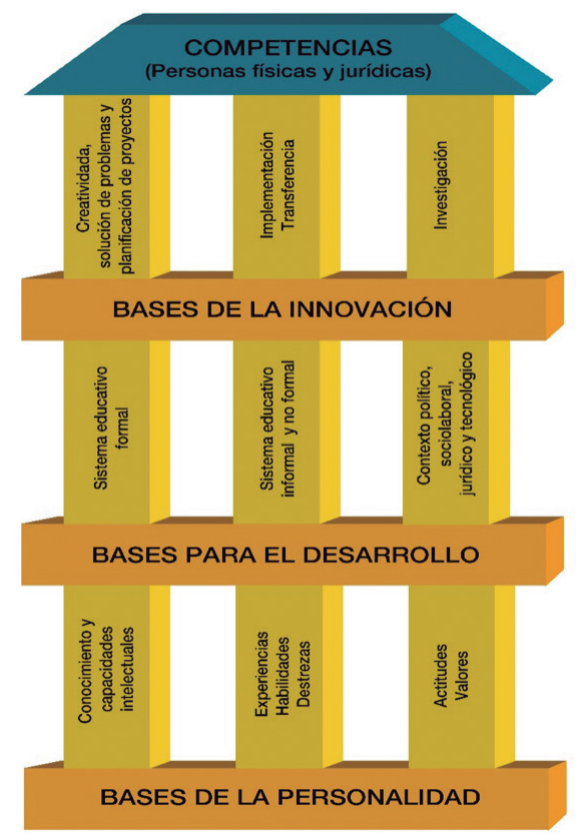

Figura 1. Bases para el desarrollo de las competencias 
Será importante, por tanto, desarrollar políticas de dignificación social de estos sectores emergentes dada su trascendencia para el desarrollo económico, el crecimiento social y el bienestar de las personas por lo que parece lógico ir eliminando la elevada tasa de prevalencia de empleo informal a través de la incorporación de estándares de cualificación profesional que nos llevan una vez más a poner en valor las competencias (Informe CES, 2018).

Es importante, por tanto, dedicar una mayor atención a revisar cómo se están gestionando en nuestros sistemas educativos las competencias cuando se sigue enfatizando la interiorización de unos conocimientos que caducan y dejar de ser útiles o desaparecen a corto plazo. En cambio la capacitación en la resolución de problemas o el hacer frente a situaciones similares en distintos escenarios o el realizar análisis con múltiples fuentes documentales o el asumir diferentes puntos de vista en entornos de trabajo colaborativo no se extinguen porque se adhieren a las personas al integrarse en su personalidad. Ser capaz de, es haber crecido, ser capaz de, es enfrentar la realidad de manera diferente, ser capaz de, es generar ideas creativas para solucionar problemas, ser capaz de... este planteamiento hace necesario interpretar la formación como un proceso de crecimiento compartido y no como un proceso de acumulación individual.

Abordar las competencias desde esta perspectiva conlleva tener en cuenta las bases de la personalidad, junto a las posibilidades de desarrollo y orientarlas hacia las competencias más humanas vinculadas a la innovación lo que supone una revolución en los procesos de aprendizaje-enseñanza, revitaliza los ecosistemas de formación pluridisciplinares como una alternativa viable y sostenible y avala el cambio cultural en las personas físicas y jurídicas concurrentes.

\section{Reflexiones y recomendaciones como síntesis final}

Europa se enfrenta a un momento de transformación que no tiene precedentes, las crisis socioeconómicas cíclicas ponen de relieve las debilidades políticas, estructurales y culturales en la UE para afrontar cambios culturales de hondo calado. El marco normativo hace tiempo que contempla los valores e ideales necesarios para desarrollar procesos de cambio bajo nuevos enfoques (Europa 2020, Agenda 2030), sin embargo, estas intenciones no se trasladan a la práctica como sus promotores esperan $\mathrm{y}$, en muchos casos, se vuelven excluyentes por competitivos. Por esa razón vamos a recordar algunas lecciones aprendidas desde la experiencia (Fullan, 1990, 1991, 1993, 1998, 2001; Escudero, 1999; Álvarez Arregui, 2017, 2018, 2019) que pueden ser útiles para transitar hacia el futuro desde otros referentes. Así las universidades:

- Deben adoptar una perspectiva ecosistémica, hacer partícipes a las personas en la construcción de la estrategia (strategizing) y tener presentes las aportaciones de autores que ya exploraron estos campos (Doyle, 1977; Bronfenbrenner, 1987; Tikunoff, 1979; Bunge, 1980; Bertalanffy, 1982; Gimeno Sacristán, 1998; De la Torre, 1998; Álvarez-Arregui, 2002, 2017, 2018; y Álvarez-Arregui y Rodríguez-Martín, 2014, 2015).

- Deben valorar la gobernanza como un referente fundamental por lo que la gestión institucional pasa por reconocer que la información y el conocimiento que generan se mueve en la complejidad de ahí que las personas que ejercen tareas de dirección, liderazgo y coordinación deben ser capaces de relacionar, contextualizar y globalizar de manera secuencial y simultánea en distintos planos lo que requiere un aprendizaje sistémico.

- Deben saber que cada institución es en sí misma un ecosistema de innovación donde cualquier persona y equipo pueden convertirse en un agente de cambio.

- Deben prever que, cuando los agentes educativos encargados de desarrollar las estrategias no visualizan que participan de la misma, serán ellos mismos los que demandarán los cambios en la gestión, en los sistemas de participación, en la búsqueda de la financiación necesaria, en los modelos de contratación o en las metodologías a utilizar.

- Deben recordar a los equipos rectorales, decanales, docentes y de administración y servicios que en las funciones que aparecen definidas en el marco legislativo se especifica claramente que el desarrollo de sus instituciones, de los profesionales concurrentes, de sus comunidades y de los usuarios depende en gran medida de cómo se despliegan sus visiones, políticas y estrategias.

- Deben ser conscientes que la capacidad de influencia de las personas que ejercen liderazgo se asocia a la utilización de los recursos (dinero, información, materiales, espacios, tiempos, etc.); su posición en los organigramas; su carisma (rasgos físicos, relacionales o comunicativos); sus valores (credibilidad, qué dicen y qué hacen); su capacitación (trayectoria en gestión, docencia e investigación); la ocasión de intervenir (sobre la base de los respaldos obtenidos en su acceso a los cargos); su estilo de liderazgo (consideración individual, estimulación intelectual, delegación de liderazgo, proyección institucional y orientación comunitaria) y su ética (mejorar la calidad de vida de las personas implicadas).

- Deben promover modelos de seguimiento y evaluación transparentes y formativos para generar confianza en los promotores y en los procesos ya que bajo este enfoque se favorecerá la comunicación, la extensión del liderazgo, la coordinación y la construcción de una visión conjunta que permitirá sentar las bases de una cultura organizativa favorable al aprendizaje continuado, al cambio, la mejora y la inclusión.

- Deben destinar una parte del tiempo y esfuerzo hacia la integración de las diferentes subculturas ya que se reducirá el riesgo de que se produzcan quiebras irrecuperables entre ellas y las personas irán participando de la visión y de las estrategias institucionales.

- Deben comunicar la visión en diferentes momentos para sensibilizar a la comunidad y deben hacerlo situacionalmente de arriba-abajo, de abajo-arriba, horizontal y transversalmente si se quieren aunar esfuerzos y fortalecer iniciativas de cambio.

- Deben abordarse los proyectos administrativos, curriculares y de investigación con una mirada transdisciplinar, ecoformadora e intercultural,

- Deben recordar que el cambio es un viaje permanente, que no se puede mandar lo que debe hacerse, los problemas son una fuente de aprendizaje, que la visión se va construyendo entre todas las personas, que el individualismo y el colectivismo tienen igual poder, que ni la centralización ni la descentralización funcionan aisladamente, que cualquier persona puede convertirse en un agente de cambio, que las culturas organizativas internas tamizan las prescripciones externas, que las relaciones internas y/o externas pueden ser críticas, que la gestión del conocimiento organizativo es compleja, que las adaptaciones 
miméticas de las directrices externas se estandarizan, no cristalizan y se burocratizan; y que la situación empeora cuando hay una alta desafección de las personas con los proyectos y/o cuando los usuarios no se identifican con las propuestas externas o institucionales ya que estas y otras cuestiones se acaban traduciendo en una pérdida de confianza y credibilidad en los programas, en las instituciones y en el sistema.
La experiencia también nos indica que, ante una realidad compleja por multidimensional las soluciones simples, excluyentes y estandarizadas ya no son viables. En nuestro caso abogamos por el desarrollo de proyectos pluridisciplinares singulares que incorporen en su diseño, implementación y análisis del impacto a los diferentes agentes del sistema político, jurídico, económico y académico. En este escenario compartimos aquellas iniciativas que profundizan en el pluralismo estructural desde los enfoques interpretativos, críticos y complejos superando las limitaciones derivadas de los modelos burocráticos, competitivos y estandarizados, para ir integrando lo mejor de lo público, lo privado y el voluntariado a través de una mirada ecoformadora, sistémica, transdisciplinar, emprendedora, ética, transparente e inclusiva. 



\title{
The evolution of the University in the Learning and Teaching Society: The value of skills in professional and personal development
}

\author{
Emilio Álvarez-Arregui (*) \\ University of Oviedo (Spain)
}

Emilio Álvarez-Arregui is professor of the University of Oviedo in the Department of Education Sciencies, currently part of the institutional team as director of the Area of the Institute of Research an Educational Innovation. He has been principal researcher in regional, national and international projects and is currently working on the development of competency models with universities, research institutes, companies, administrations, counseling, social agents and platforms for accelerating innovation from different American and European countries. It has more than two hundred publications in magazines and books relative to these and other topics. He has made teaching and research stays in Spanish universities (Seville, Huelva, León, Balears Islands, Jaén, UNED...) and foreigners (Uruguay, Chile, Latvia, Portugal, USA, United Kingdom, Ireland, Brazil, Switzerland...). He is member of "the Spanish Society of Pedagogy", the "Universities and Inclusive Education" Group, the "Spanish Service for the Internationalization of Education", of the "European Network of Inclusive Education and Disability Included"; "European Forum of Education Administrators", "Transdisciplinary Educational Research Network"; "Inclusive University Network, Network of Educational Research Institutes of Spain", "Alfamed Euro-American Network"; "Ibero-American Network for the Evaluation of the Educational Quality of Higher Education"; of "the University Network for Postgraduate Studies and Permanent Education"; and "the UNESCO Network in the Principality of Asturias", among others. He is director of the "Aula Abierta" magazine.

ORCID: https://orcid.org/0000-0002-4657-753X alvarezemilio@uniovi.es

\section{ABSTRACT}

Society, Education and the University have been interrelated based on the economic, social, structural, technological, political, legal and functional determinants of each historical moment. The decisions adopted in this ecosystem have been, are and will be controversial because they are not ideologically neutral, which has ended up determining organizational and functional cultures linked to the current systems of power and knowledge that are legitimized in the geographical areas in which they are integrated.

In this article, we take a look at the past, present and future of the university from different perspectives that support the need for these organizations to evolve from a systemic and multidisciplinary approach, which requires aligning learning, teaching, training, research, management, talent and commitment from an integrating paradigm that allows us to generate the necessary innovations to adapt and project ourselves into an environment that has no previous history and whose evolution we are unaware of. Consequently, it is necessary for the university to assume the real value of the competencies so that the people who attend these institutions learn and teach how to learn and undertake if they want to adapt to a context of fast and complex change.

Keywords: learning, teaching, training, research, competency and educational innovation.

\section{Contextualization}

University, as an educational institution of reference in Higher Education, has adapted to the economic, social, structural, technological, political, legal and functional determining factors of each historical moment. The decisions adopted have been, are and will be controversial because they are not ideologically neutral, because they affect institutions differently and because they do not discriminate against the singularities of the participating groups. This situation has generated organizational and functional cultures linked to the current systems of power that have legitimized it socially, politically, legally, academically and scientifically, which has had consequences of different prognoses in its own fields and environments.

Today's society is being interpreted as a glocal ecosystem where global and situational actions are simultaneously generated and used to move towards an economy of data, information, knowledge and wisdom in a Society of Learning and Lifelong Learning. The problem that is being detected in the decisions that are being adopted is that they are giving priority to short and medium term decisions without sufficiently valuing their long term consequences. Nor does it seem that there is a desire to understand that structural changes are not accompanied by cultural changes, which is generating deviations between the intentions of the promoters of innovation and the results obtained. From there, it is necessary to clear up unknowns in multiple dynamic equations in order to redirect the emerging debates that confront administrators and the recipients of education services. By way of introduction we incorporate some questions that are on the game board of university organizations currently, namely:

Are university teachers prepared to transmit the principles, values, attitudes and skills that are demanded as necessary from the political, academic, social and work environment in their cultural reference environments?

To what extent can these institutions develop competencies such as social skills, leadership, teamwork, stress management, emotional intelligence, discipline, responsibility, commitment, productivity, predisposition, continuous updating, loyalty or systemic thinking in their training cycles? 
Is it realistic to continue presenting university certifications as sources or indicators of user skills, stability, selection, status in the community and social, professional, cultural, political and personal development?

Is it feasible and/or necessary to design a universal curriculum to facilitate personalized learning for all people, guarantee equal opportunities, promote multidisciplinary work or encourage collaboration based on competition and standardization?

Is it possible to reorient individualism, credentialing and bureaucratization towards collegiality, teamwork, autonomy, flexibility, creativity or innovation with the current legislative framework?

Is social responsibility really valued from the academic, social, political, trade union and business spheres on the management, resources, financing and selection of personnel in the university or are the internal power groups and their interests the ones that guide the processes?

How can the reactions of large corporations to the monopoly of university certification be affected?

Can dual training generate more solid bridges between business, academia, social agents and the political-legal environment in a generalized way, should it be approached in a differential manner, should it be given a multi-sector approach

Do the systems of initial, access and continuous training respond to the professional needs of university education agents?

Should new times for lifelong learning and family reconciliation be negotiated with the social partners at global, European, state, regional and institutional level?

Where is the creativity, the solution of problems, the development of projects, the control of emotions and the impact of knowledge on the end users?

What cultural changes has the 2020 Strategy generated in the university sphere and how is Agenda 2030 interpreted?

These and other issues being discussed in different forums are subjecting the socio-educational ecosystem to multiple pressures, as endorsed by international, national and institutional reports (CES, 2018) in which they warn of the loss of effectiveness of formal education systems. This first reading already points out that a model that was valid in previous times is repeatedly questioning the exponential increase in demands for change that forces individuals and corporations to rely on permanent innovation and continuous multidisciplinary learning and teaching in all areas as an adaptation strategy, but that does not find an echo in Higher Education institutions (Álvarez-Arregui, 2017, 2018, 2019).

In the following sections we provide different interrelated views of the university with the intention of helping us to understand the evolution of its missions and thus have a greater number of references to build a systemic, systematic and flexible vision that allows us to project ourselves towards an uncertain future by strengthening meeting points, negotiating dissensions and endorsing the processes of convergence and divergence to address problems and deploy projects. This approach requires aligning learning, teaching, training, research, management, talent and commitment from an integrating paradigm where political, academic, business and social cultures converge to generate the necessary educational innovations from which to respond to an environment that has no previous history and whose evolution we are unaware of. Consequently, it is necessary for the university to reflect on and assume the real value of the competences so that people learn and teach how to learn and undertake if they want to adapt to a context of rapid and complex change.
A retrospective look at the University: etymology, visions and models

A first approach to the word "university" takes us back to its etymological root in Latin where the term "univeršrtas, -ātis" refers to the synthesis of unus (one) and verto (turned), that is, "turned towards one". "Verto" also refers to "to pour, to spill and to throw", but also to "versar" which is to turn something upside down, to deal with a matter; hence someone versed has been associated with someone enlightened. Moreover, if we consider that verse comes from the universe, the whole, the totality, what surrounds it (unus, versus) we find that University refers to a complex object whose parts are related to at least some of the other components (Bunge, 1989: 196).

In its original use, University designated the teaching group (universitas magistrorum) and, later, the student body (universitas scholarium) sharing roots with universe and universal. Therefore, university would be the point where everything unites and turns, it is a totality of studies and scholars that move and change together (López-Gómez, 2015). This unity will be linked to community (communitas) so the university would become a constant search in common-unity and universitas would be identified with a corporation or community oriented towards a common goal where the genitive determined the nature of the space where its members have common interests so they acquire their own and independent legal status and where the transmission and production of knowledge would be consolidated as the first mission of these institutions (Zonta, 2000).

The medieval university will be nourished in its origins by plural references (Greek, Latin, Christian, Jewish and Arabic) creating an adequate context for the cultivation of knowledge which will identify them as hermetic "ivory towers" although their value as a focus of social and community development will always be emphasized. As is logical, their development will not be homogeneous and two approaches will emerge, one more horizontal - broadening of knowledge - and the other more vertical - deepening in each field of knowledge- which will derive in the long run in two positions, one more humanistic, associated with a generic knowledge, and the other more specialized, linked to a more specific scientific knowledge (López-Gómez, 2015).

Snow (1987) will point out on this argument the emergence of "two opposing cultures", the scientific and the intellectual, which will favour the modernization of the university through the dissection of the knowledge that is the fruit of scientific specialization; through the medieval reorientation of teaching and training towards research and academia; through the progressive distancing of the university from the Christian tradition; through the growing approximation of the institution to the state; and through the recognition of the basic academic principles of freedom of thought and teaching freedom.

The Renaissance, the Enlightenment, the First Industrial Revolution, the creation of the Nation-State in the 19th century and Humboldt's directives in Berlin were other important reasons that led to the emergence and consolidation of the second mission of the university, research, which was to be incorporated unevenly into the European and American models that developed (Álvarez-Arregui, 2010c).

The modern university thus evolves as a "circus" with several tracks where research - Humboldtian model-, training of professionals - Napoleonic model- and intellectual education of a training university - British model- are presented as three basic references. These models will be revised after the Second World War as the Humboldtian ideal loses its validity and the elitist 
university is questioned in the face of the massification of higher education (1960 - 1975) as a result of economic development; the increase in the average standard of living, the rise in the birth rate and the commitment of governments to satisfy citizen demand (Trow, 2007). In any case, this expansion will be revised downwards since 1975 due to the strong increase in public expenditure generated by the creation of new universities and by the economic crisis.

In the last decades of the past century the university question will be considered as a problem of society as a whole from the point of view of theory, but in practice there will be talk of a Technological University (OECD, 1999) that is constantly evolving. In this respect, it is worth remembering how the notion of 'triangle' that explained the interconnections between university, government and industry led to the notion of the 'triple helix' where each one of them integrates functions from different fields (Etzkowitz and Leydersdoff, 1997). This change of approach will extend the horizon of this institution as it was reflected in the Report University 2000, Bricall Report, when it was endorsed the importance of establishing bridges of union with the social and enterprise surroundings, fact that although it questioned the German model would be ratified in the Tunning Report (González and Wagenaar, 2003: 248). This new vision will not be generated by chance but as a result of the concurrence of several macro trends that were consolidated since the end of the 20th century in an interdependent way, we refer to glocalization (Castells, 1999, 2000), neoliberalism (Bell, 1991; Gimeno Sacristán, 2001) and to the exponential scientific-technological development (Dosi, Freeman, Richard, Silverberg and Soete, 1990). The repercussions of the new framework have not been long in coming and are causing new economic, political, legal, labour and social models to germinate, obliging individuals and companies to adapt permanently to change, which is necessarily transferred to university institutions that are being forced into a process of continuous restructuring and re-culturation that is having differential effects depending on the histories, strategies and financing capacity of these institutions (Álvarez-Arregui, 2017).

The third mission of the university thus emerges as a logical consequence of a socio-labour environment that seeks to strengthen entrepreneurship, innovation and social commitment as its mainstays (Bricall, 2000; Álvarez-Arregui, 2018). This institution will thus assume a more managerial approach by asking it to be an excellent organization that has to contribute to the economic and social development of the area in which it is located by competing, collaborating and associating with companies, institutions, associations and technology centers in the regional, national and international spheres, but also to expand civic and human values (Álvarez-Arregui, 2019).

The increased demands on the University have translated into a wear and tear that is manifested in different types of discontent that has been greater or lesser depending on the geographical areas. In the case of Spain, the response has not been long in coming, and at the Conference of Spanish University Rectors (CRUE) on 22 April 2019, a decalogue of requests was presented to the political parties to improve Higher Education if these issues are to be addressed with minimum guarantees of success. Among other demands, this document called for a new law on universities, sustainable financing, an increase and stabilization of staff, improvement of teaching, support for research, transfer and innovation, defence of equal opportunities and student rights, improvement of management, promotion of internationalization, transparency and accountability. In this scenario, multiple debates have opened that demand arbitration between administrators and education administrators since all parties must have a stake in the outcome. It is thus necessary to construct a BPANA (acronym of Best Possible Alternative to a Negotiated Agreement)(Fisher, Ury and Patton, 2002) from a critical glocal reflection and with a roadmap, Agenda 2030, which has been presented in our cultural environment as the compass that indicates the path to follow and the content of the debate in the coming years.

\section{A critical look at the current situation: standardization and quantification}

The response capacity of universities will be constrained or favoured situationally based on how the normative framework is articulated so that governments have an important share of responsibility when they assume public commitments, when they make insufficiently informed decisions, when the initiatives they want to promote do not translate into practice as expected, when intentions are not reasonably explained, when short-term policies are promoted or when the values they transmit are not derived from the ethical principles they claim to defend, among other issues.

In the light of these issues, Europe has been reviewing its university model from a socio-political perspective, placing emphasis on the economy, working conditions, skills, social balances or territorial developments at different times. The effects of the decisions taken have meant a global redesign that has affected all universities in general, although it has had differential repercussions on countries, institutions, professionals and users. Proof of this are the positions reached in the rankings of universities, so in the 2019 Shanghai and earlier rankings we find lights and shadows based on the results achieved in our environment since the United States systematically dominates the world ranking year after year. As a reminder, it currently ranks 17 universities in the top 20, Harvard (1st), Stanford (2nd), MIT (4th) and Berkeley (5th) and 120 universities in the top 500 worldwide. In Europe, the United Kingdom ranks 34 (8 in the top 100), Germany 27 (4 in the top 100) and France 21 (3 in the top 100), which informs us of a verifiable weakness of the EHEA in Higher Education in terms of worldwide research excellence in countries that could be called leaders.

These differences can be interpreted as logical if we take into account the levels of development and investment made by the different countries, so it would be expected that distances would increase if not change the trends that are consolidating as the recruitment on campuses and / or in American research centres that have hosted more than 400,000 thousand researchers trained in Europe in recent years. In this scenario, it cannot be ignored that in the Asian area, China, India and the Middle East continue to maintain and/or increase investment in their university institutions, thus increasing the offers to researchers to help improve these rankings, which capitalises or decapitalises the institutions in terms of the highly qualified human resources available and enters a knowledge management market. If we focus our attention on our environment, we see that Spain places 13 centres among the top 500 institutions: The University of Barcelona (174), the Autonomous University of Barcelona (202), the Complutense University of Madrid (209), the University of Valencia (291), the Autonomous University of Madrid (302), Pompeu Fabra (398), the University of Granada (366), the Polytechnic University of Valencia (444), the University of Oviedo (481), the University of Seville (485), the University of the Balearic Islands and the University of the Basque Country (401-500). 
Although there are many readings that can be made, we are going to refer to those that are incorporated from the political key, so the government of Spain indicated that we have 47 public universities that teach face-to-face and of these, 38 are among the 1,000 best and only the private University of Navarra is among them, which is justified as an oversight or disinterest in research and knowledge transfer from these entities. This presentation of the data is problematic because it is subject to ideological reinterpretation in different forums, especially when neoliberal globalization is identified with Higher Education or when it is indicated that the current situation is the result of a pre-designed strategy of the knowledge economy within the new paradigm of Public Management where market policies, the obtaining of economic benefits from the public, or the leadership of knowledge in universities is evident (Saura and Bolivar, 2019). In any case, we must also be aware that the results may vary if other measurement indicators are included, such as the quality of training, the employability of graduates or the real transfer of knowledge and services/products that users incorporate from innovation, something that is done when policies and investments are to be justified (Álvarez-Arregui, 2017, 2019).

In light of these arguments, it is clear that higher research institutions and universities that promote R\&D\&I are globally affected by a structural neoliberalism that determines different stages of institutional development that require differential treatment in terms of the strategies they promote to project themselves into the future but taking into account the rest of the macro-variables and the stage of evolution of the institutions. Although we have already referred to this issue on other occasions (Alvarez-Arregui, 2010, 2017, 2018 and Alvarez-Arregui and Rodriguez-Martin, 2015) we have made a classification, could be another, of university institutions to support our arguments, so we distinguish between:

1. Organizations of Excellence and World Reference (First level). This is where the top 100 organisations in the world with consolidated multidisciplinary projects and strategies in constant development with regional, state, international and global projection would be located. They have met the quality standards for years without problems.

2. Organizations of International Excellence (Second Level). Here we would place the organizations between the positions 100 and 500 of the rankings. These institutions try to maintain or progress in this space by designing and developing integral and international visions and strategies with wide diffusion, a stable financial coverage and a policy of strategic alliances. In these cases, the institutions do not meet all the standards but have a positive evaluation in most of them. The University of Oviedo would have managed to join this group by the end of 2019, something it had not achieved since 2003.

3. Regional Reference Organizations with International Projects (Third level). The fork could be extended to the 1000th position. These organizations have prestige in their reference contexts and are valued internationally in some areas. They have a vocation to develop multidisciplinary projects with regional, national and international repercussions. They have difficulties in meeting their own standards and invest in strategic networks to try to improve their position.

4. Organizations in search of their Excellence (Fourth level). This would include those organizations located from position 1000 onwards. Obviously, the range of institutions that can be found in such a wide range is very wide and should be subject to greater segmentation, although in this case it is used only for explanatory purposes. Here we find young universities, or those that are in the initial stages of development or that have difficulties in financing or that do not have the necessary structure to develop or are satellites of other universities or are developing peculiar projects independently of the rankings.

Although this categorization would be an approach to a complex reality because it is multidimensional, it allows us to remind educational administrators to be cautious when making comparisons because it is easy to make biased readings and they should be aware that when they make statements about their institutions to improve in the rankings they "forget" to indicate that the emergence of hundreds of universities in the world, in the case of China, will progressively displace European institutions due to the greater financing and contracting capacity of their governments.

With regard to ownership, we could understand a support to private universities under the condition that they reinvest in the institution itself or in its nearby areas of influence the benefits they obtain or an important part of them, in the case of Harvard or Princeton, but we will not endorse those formulas that distribute benefits among shareholders since when the profit motive is a priority, the quality of education and equity begin to be questioned because the right to education and equality of access, something that the states claim to guarantee from their constitutions, are put into question.

Nor can market criteria orient all curricular offerings toward the most demanded degrees or guide the selection of students through their economic capacity with standardized curricula; rather, they must develop well-founded and designed medium- and long-term policies. Nor does it seem viable that the exploitation of the results derived from knowledge should be done independently of regional development by technology parks or business incubators (spin-offs), since then there is a risk that research will be oriented toward short-term profitability, mass for critical reflection will be lost, social responsibility will diminish, disappear or be masked, and access to a universal transversal higher education curriculum, at least in the degree, will be questioned.

With regard to the rankings we must also be aware that the lists focus on the 500 or 1,000 universities of the almost 27,000 university centres that exist in the world, which means a hidden invisibility of institutions, professionals and students affected as well as the variety of situations they face. This is why education administrators should let the public know that the assessment criteria contain subjective components (prioritization of indicators, salaries, time to complete studies, graduate, master's and doctoral rates, teacher-student ratio, teacher selection systems, employability rate...) and questionable components (measuring teacher quality using data such as the number of Nobel Prizes obtained by their students, productivity, bibliometric indicators, importance of language, location, access...) (Álvarez-Arregui, 2019)

Nor can education managers continue to ignore the lessons learned about organizational development and cultural change in organizations. By way of example, we will recall Senge (1990) when he pointed out three decades ago a reality that continues to be present in the university environment. In the words of this author, the true commitment to the strategies promoted in organisations is not usual, which is why their promoters develop 
a transactional leadership where a short-term vision is "sold" to obtain specific support, but they will not obtain real and sustainable support since a style of distributed transformational leadership is more appropriate for this (Álvarez-Arregui, 2002).

In the university institutions we also find groups that assume an uncritical enlistment that supports the vision but that ends up being incorporated routinely in the processes because they do not feel they are co-participants in it. Senge (1990) also pointed out in this respect the need to differentiate between genuine compliance and commitment, since the former goes further in terms of the energies and effort that a group or collective is willing to commit to achieving the vision. In the face of disobedience and apathy, this author advised us to focus on listening and hope from a realistic point of view, which would translate into situational leadership as a result of a deep reflection on the reasons given. In these cases, it is necessary to be aware that the best thing is to speak clearly, avoid hypocrisy or generate false expectations because otherwise confrontations, hidden blackmail or open conflicts will emerge. In the face of excluding radical positions, little can be done, and it will be the regulations that mark the decision to be taken.

The faces that are being shown inform us that the path to be followed by higher education institutions will not be easy but will be even more tortuous if they continue to be required to join systems in which they have to climb "fictitious" positions under the backing of governments and companies that are more concerned with maintaining the current system than with the needs of the citizenry. Faced with this situation, it is necessary to remember some omissions that are not usually communicated, and of which we highlight the following:

- the career is uneven because not all institutions will be able to integrate into the highest levels of excellence because the starting point is not the same, there are different routes and they can change in unexpected ways,

- the stages of institutional development are not equivalent because the histories of universities have generated unique organizational cultures that condition them,

- widespread competition will continue to encourage individualism, anxiety and stress among the agents involved,

- knowledge is becoming unattainable in view of the development of communication and the multiplication of production, distribution and control centres, which will make it difficult to select what is relevant to the accessory on multiple levels,

- teaching and research teams can design, innovate, apply or experience their degree of excellence or failure independently from the rest of the institution, which has led to an ongoing slowdown in collaboration, training and sustainable development,

- the financing capacity is fundamental, so the budgetary base and its sustainability have differential impacts of different prognosis if we want to tackle the strong endogamy of the system, the ageing of the workforce, the precariousness of some groups, the lack of resources to retain talent, or the urgent need to harmonize State policies with those of the Autonomous Communities in Higher Education in the case of Spain and

- the paradoxes, contradictions and inequalities are difficult to predict because by becoming part of the organizational cultures it could break the ideals of an EHEA which, although it has followed the indications of the Tunning Project to translate the available knowledge into viable knowledge from the economic and commercial point of view, has many deficiencies in professional, institutional and citizen development

\section{A hopeful glance}

The dynamics that have been established in the relations between Society, Research, Training and Innovation are a challenge for the university institutions of the future. These organisations will be able to adapt to the new challenges when they understand their own histories, when they take into account their contexts, when they give a voice to the participants and when they develop visions, missions, strategies and actions that combine restructuring and reculturization simultaneously, taking the references of the Agenda 2030 as the unifying axis.

The university institutions have to be aware that the digital revolution has changed the rules of the game because it is generating an accumulation of information and knowledge of such magnitude that it forces to modify again and again units of measurement to be able to follow the rhythm of production, thus it has passed from Kilobyte (KB) to Megabyte (MB), to Gigabyte (GB), to Terabyte (TB), to Petabyte (PB), Exabyte (EB), Zettabyte (ZB) and Yottabyte (YB) which entails developing new data, information and knowledge management systems to develop teaching, research and transfer which has revitalised the Big-Data or more recently the Blockchain but has not taken into account the cultural and formative change that this fact entails. Other problems added to this hyperproduction of knowledge is that they come from multiple fields, are volatile and highly fragile, which demands a review and redesign of the nature of the learning and teaching processes in terms different from those of previous societies.

The university of the twenty-first century is faced with the task of reorienting its focus from transmission to the generation of learning and teaching contexts. In the case of university institutions, new forms of learning, teaching, content selection, implementation, application and organization that generate significant knowledge for the planet, for professional and living environments and for ourselves must be rigorously explored (Álvarez-Arregui et all. 2017). In this regard, we agree with Edgar Morin (1998: 24) when he points out that the basic function of the university is to promote the reform of thought in order to make people capable of creating, relating, contextualizing and globalizing, in short, that they have complex thought.

In short, the university must prepare active citizens who are good professionals (specialized), but it must also train polymathic people (multifaceted, multidisciplinary) with a mind that allows them to reflect and analyse in a systemic way the world that surrounds them, working in multidisciplinary teams to adapt and give answers to the problems that emerge in the social, economic, political, labour, cultural, leisure and personal environment.

In this context it is worth taking up again some normative milestones that have been referenced until now, the first being the Maastricht Treaty (1992) where the political bases for higher education in a new Europe were established, The second was the PISA Program that updated the previous one through the periodic records of academic and professional profiles and the third was the Tuning Project that opened a new educational debate with the intention of identifying and exchanging information and promoting collaboration in the European space for the development of quality, effectiveness and transparency (González and Wagenaar, 2006). Finally, the Sorbonne Declaration 
(1998) promoted the alignment of governments to harmonize European higher education. The European recommendations that emerged from these milestones have not been implemented as expected because countries and organizational cultures have sifted through the prescriptions, leading us again and again to paradoxical and contradictory situations. It is logical, therefore, that in the current scenario what Lawn and Grek (2012) call "chaotic uniformity" is taking place where European higher education is sailing without a clearly defined course as it tries to preserve in practice the diversity of European university cultures.

The Agenda 2020 has tried in the last decade to address these problem situations that have been generated by focusing on the generation of a Platform of "Smart Specialisation Strategies" (S3 of the European Commission) by openly raising the importance of regional policies for smart growth. This has been done by assisting Member State authorities and regions to optimise the impact of regional funding from the so-called knowledge triangle: Education, Research and Innovation [COM(2010)546]. In this process, although attempts have been made to mobilise universities in support of regional development from a transformational approach, the results being obtained show both light and shade. A new vision has therefore been projected from Agenda 2030 to revitalise once again the role of universities by assigning them a key role in all the phases on which their fulfilment depends at international, national and local levels.

This is how it is proposed for the next decade that the University should generate, transfer and disseminate open knowledge in the service of society, well-being and sustainability, since it is assumed that it has the necessary capacities to make a critical and constructive contribution to sustainable development based on its values and knowledge. Therefore, these institutions should put their influence at the service of global sustainable development, not only with respect to ODS 4 (now a quadrilateral), but also to the 17 objectives set out, in which inter- and transdisciplinary research (innovations and solutions, local and national implementation and capacity building in this field) stand out; Education (for sustainable development, jobs to implement ODS, capacity building and mobilizing and involving youth; Management and Governance (aligned with ODS) and Social Leadership (public commitment, action, intersectoral dialogue, policy development and advocacy towards ODS, mobilization and positioning of the sector towards ODS and engagement of the university sector).

The "official" model adopts an entrepreneurial position by attempting to efficiently combine the interests and initiatives of the Public, Private Commercial and Third Sector by applying to Foundations as possible poles of talent attraction. More heterogeneous organisational and functional charts are recommended, governing boards that incorporate external members elected / represented in collegiate bodies (Senate or Academic Senate) and/or the corresponding Ministry. The Governing Board would appoint and dismiss the rectors but would also help determine the mission, vision and strategy. There is also a need to strengthen institutional management, linking funding to development, performance and impact. Reduced regulation would be correlated with increased monitoring, evaluation and accountability to the relevant authority. On the other hand, the need to strengthen the executive and coordination bodies is pointed out, so that the rector could appoint not only his management team but also the deans, who would designate the directors of the departments and research centres. In the case of the management teams, this entails training in institutional management, leadership and social responsibility from the moment that they are going to work with public and private resources from donations, patronage, associations with companies, creation of spin-offs or through the differential services that are determined.

These proposals are the object of multiple debates and nuances and therefore will not be easy to implement given that they implicitly imply a radical change in the way of understanding university institutions as we know them, which makes it necessary to address each case in terms of the meaning and value of the knowledge it generates, and the perverse effects of the disregard for cultural diversity, to the different possibilities of access to financing, to the effects of the participation of the business environment in the construction of the curriculum, in the case of dual training, to the precariousness of many employment contracts, to a growing standardization of curricula that makes inclusion and personalized learning difficult, or to the increase of individualism due to excessive competitiveness.

Before closing this section, we believe it is necessary to remind those employers who question the qualifications of university graduates in initiative, responsibility, availability, capacity to adapt to change, geographical mobility or development in social situations,... In other words, this group places special emphasis on the moral dimension of work because it detects that the attitudes of university graduates do not show a commitment or capacity for sacrifice when they enter the work environment, which enters into another neuralgic point that will have to be debated in the coming months: the teaching of values, of knowing how to be, something that has been displaced in the teaching-learning processes in recent decades (Álvarez-Arregui, 2018).

In view of these initiatives, the arguments presented and based on our experience, it should be pointed out that, in our opinion, improving the university system no longer involves associating the development of higher education institutions with macroeconomic variables or thinking, when there are difficulties, that we are at the bottom or middle of an economic cycle, but that we have to decide where we want to direct the available resources in a sustainable way to tackle and overcome a crisis that is structural, functional and cultural. This approach requires an in-depth review of the skills that should be generated from the university, but this requires a real participation of the academic, political-union, business and social spheres because they all have an impact on education and social responsibility is shared.

\section{Competencies as a benchmark in a Learning and Innovation Society}

Society has always valued the qualifications of its members in one way or another, so traditionally the work that provided people's livelihood was acquired through skills that were acquired outside formal education. Logically, this situation evolved until people were considered competent to carry out work when their unique knowledge and behaviour allowed them to perform a certain function in social, labour and educational organisations or on their own account.

This way of understanding competencies that has been maintained over time has been questioned in recent decades as it has become increasingly visible that in the coming society knowledge will be necessary but not sufficient to perform the functions in the personal, social and working environment. A high degree of adaptability will also be required to adapt to a highly dynamic environment, which is more supportive 
than in other times of systemic knowledge, multidisciplinary collaborative work, lifelong learning and innovation.

The problem that this process of change generates in developed countries is evident when the demands on workers are increased, wages are stabilized, jobs for life are questioned, atypical jobs appear as effects of scientific and technological development, or production centres are relocated, among other issues. It is logical that professional wear and tear, burn-out syndrome, increased demotivation, rust syndrome, marginalisation, exploitation and exclusion find their breeding ground here. The exponential growth of robotization and digitalization is another faithful reflection of the emergence of an artificial intelligence that makes it necessary to promote at all levels new forms of learning and teaching that combine, provide feedback and integrate basic, generic, digital and personal skills to know, know how to do and know how to be a cosmopolitan citizen.

Policies seek to balance emerging imbalances but to be effective they must be equipped with an eco-systemic vision of the short, medium and long term future if they are to anticipate the future by preparing citizens for change. The actions to be taken include redesigning the current models based on situational diagnoses that are attentive to social, work, cultural, leisure and personal changes in order to develop initiatives that improve the skills and qualifications of individuals and companies, but also to strengthen and increase synergies between the academic, trade union, business and social spheres when they work on shared projects.

The EHEA has already highlighted in its principles the importance of competence-based training as formalised in different standards in the different states. In Spain, this was reflected in RD 1393/2007, which indicated that "the curricula leading to the award of a degree must (...) have at the centre of their objectives the acquisition of skills by students, extending, without excluding, the traditional approach based on content and teaching hours. Emphasis should be placed on the methods of learning these skills, as well as on the procedures for assessing their acquisition". The implementation of this approach has many difficulties because the competencies are "complex structures of processes that people put into actionaction-creation to solve problems and carry out activities of daily life and of the labour-professional context, oriented to the construction and transformation of reality" (Ruiz Corbella, 2006: 100). In addition, in this training system reference was made not only to a merely formative dimension (concepts and procedures) but it adopted a more educational dimension by incorporating axiological and attitudinal questions together with the contents. In this regard, although we share the idea that the competencies should incorporate the intangible intellectual assets that the most genuine university tradition has always cultivated, we consider it a serious error to have reduced their potential in those institutions that are diverting the value of the university towards measurement, quantification and bibliometrics. This perspective is forgetting that the process of learning-teaching of competencies requires a professional and personal cultural change that requires resources and efforts sustained over time.

The approach from teaching to learning (Barr and Tagg, 1995) or learner centred teaching has also given us to understand that the student would build his knowledge through the collection and synthesis of information in a collaborative and shared culture. Furthermore, creativity would generate the best questions to develop projects from which to investigate and understand error as learning with the intention of continuing to try and not being afraid of failure. In the end, we see that neither the first nor the second intention have become generalised, but what has become established is greater standardisation and bureaucratisation. At this point, it should be noted that neither the managers, nor the teachers nor the students have had the desirable support to equip themselves with the necessary tools to offer, facilitate and develop the skills that are reflected in the teaching programmes they have developed to adapt to the EHEA. Thus, the model focussed on learning has not been implemented in practice as was desirable, hence we will have to continue working in this direction by endorsing all those initiatives that understand that the teaching function has a high degree of linkage with the process of guidance and support of trainees in their academic and professional career at the entrance, during and exit of the system in which it has been incorporated (Álvarez-Arregui, 2017).

University tutoring now regained its full role by establishing specific spaces and times where learning and teaching processes could be established that went beyond merely resolving the difficulties derived from the programme of a subject, but as in previous cases, it did not sufficiently consider the professional and cultural change that it entailed, so that in the end good intentions that are not properly translated into practice continue to be reflected on paper. Therefore, we can talk about one more deficit, which has not been overcome, and which we will have to address through awareness campaigns that are accompanied by continuous transversal training if we want to make the demand for active participation of people a reality, understanding them as trainees as a whole.

The concept of credit, although it incorporated structural modifications, has not always been functional because by measuring the learning process in hours of teaching and effort of the learner what was done was to artificially increase the complexity of university planning, methodology and evaluation but this fact was not accompanied by sufficient investment in space, time, recognition or training so it can be said that its impact has been limited.

Once again, the problem has been the difficulty of integrating the new functions into the professional imagination and, unlike what was initially proposed, what has been strengthened is credentialing, standardisation and bureaucratisation. Therefore, it is necessary to act with caution when speaking of observable or demonstrable skills and to differentiate between a curriculum aimed at training for employment and a curriculum for professional training, since its meaning changes in its second meaning when it becomes necessary to integrate skills and not to generate aggregations. In this sense we agree with Le Boterf (2001) when he points out that one cannot work with a weak definition of competence, that is, it cannot be understood as a sum of knowledge of knowing how to do or know how to be or as the application of theoretical or practical knowledge. In most cases, competence is assimilated to a fragmented activity (...) The logic of decomposition kills competence. In our case, it seems more appropriate to define competencies in terms of combinatorial knowledge that brings together a whole set of knowledge, procedures, attitudes and skills that complement each other, so that in order to act effectively in professional situations a person must "know", "know how to do", "know how to be", "know how to learn" and "know how to do", incorporating an overview of the "types" of competency (Álvarez-Arregui, 2017). As a reminder, we present in the attached table a brief indicative scheme. 
Chart 1.

Technical and social competences (White Paper of the Degree in Pedagogy and Social Education, 2004: 155)

\begin{tabular}{|c|l|l|}
\hline \multirow{2}{*}{ TECHNICAL SKILLS } & $\begin{array}{l}\text { They involve the mastery of knowledge } \\
\text { and skills specific to a given professional } \\
\text { field }\end{array}$ & $\begin{array}{l}\text { KNOWLEDGE: General or specific, theoretical, speculative or technical } \\
\text { scientific knowledge }\end{array}$ \\
\cline { 3 - 3 } & $\begin{array}{l}\text { KNOW-HOW: Mastery of the methods and techniques specific to certain } \\
\text { professional fields }\end{array}$ \\
\hline \multirow{2}{*}{ SOCIAL SKILLS } & $\begin{array}{l}\text { They involve motivations, values, } \\
\text { relationship skills in a social organizational } \\
\text { context }\end{array}$ & $\begin{array}{l}\text { KNOW HOW TO LEARN: Capacity for continuous training at the pace } \\
\text { of developments in the professions, in technology and in organizations }\end{array}$ \\
\cline { 3 - 3 } & $\begin{array}{l}\text { KNOW HOW TO BEHAVE: Behavioural attitudes at work and ways of } \\
\text { acting and interacting }\end{array}$ \\
\cline { 3 - 4 } & $\begin{array}{l}\text { KNOW HOW TO SHARE KNOWLEDGE: Enabling learning to take } \\
\text { place in the workplace, as a result of personal intercommunication }\end{array}$ \\
\hline
\end{tabular}

A functional approach to the competences that we share is that which identifies them with the capacity to mobilize different cognitive resources to face a task in a determined context (Goñi, 2005: 87) because it associates the capacity with "something" that is possessed in potential and is manifested in act when it is needed which allows designing and orienting the training processes towards the achievement of such competence. Having made this clarification, it is necessary to distinguish as components of the competence (ob. cit.):

A mental operation (Being able to...) - mental action that has to do with personality - motives, traits and self-concept -, an object (usually called knowledge), a content (makes the object specific - conflicts, organization of spaces, etc.) and a purpose (application context). The formula under which it would be presented would be the following:

\section{COMPETENCE $=($ Mental Operation + Object $)$ + CONTENT + PURPOSE}

A possible wording of a competence would be as follows: A person capable of carefully diagnosing a situation of labour conflict in the immediate environment (municipal, regional or institutional) and assessing various alternatives to position themselves in a reasoned and responsible manner for any of them. In this definition, explicit mental operations appear (to analyse, to value alternatives and to decide) and objects on which one acts mentally (concepts and procedures necessary to analyse a conflict, the alternatives to consider and the elements that come into play to take a decision, without leaving aside the attitudes to mobilize). The proposed action has a specific purpose aimed at making a responsible decision, although further clarification is needed to help understand it.

The operation is important because it is understood as an internalized action that is carried out in a symbolic way, which allows us to differentiate the operations from the actions, since the first ones imply manipulations of symbols or mental images and the second ones imply objects of the natural world (ob. cit.). Therefore, to operate is broader than to act and it is better adapted to the term competence:

- Conceptual (nouns, noun forms). Words or terms of a linguistic nature - work, power, kinetic energy, etc. - that are linked to knowledge.

- Procedural (verbs). Operation(s) on an object. The written expression of a procedure is called protocol and is associated know how.

- Attitudinal (adverbs and adjectives). All human relationship is mediated by values, behaviours and attitudes on which one does not operate as with concepts or procedures but as regulators of operations, that is to say, one operates with it, we refer to the knowledge of how to behave.

De Miguel (2006: 32-33) addressed this issue by delving into the components of competence for which I will go into more detail:

The person's intention, where the following is taken into account:

- personality traits, understood as those characteristics that are physically manifested and involve consistent responses to situations or reactions (achieving something unique, difficult to achieve) that can be subject to different degrees of intensity, what we call regulation

- motivation, associated with what the person thinks or wants as a cause for a certain action (going to class, participating, doing a practice, preparing an exam) and does it to achieve goals (passing a subject, mastering a skill, meeting a personal need - belonging to a group)

- self-concept that will reflect attitudes, values or one's own personal image - predisposition to leadership, value placed on qualifications or inclination to collaboration and, finally

knowledge will link it to the information available to a person on content areas that may relate to concepts, facts and procedures.

Action - behaviour

- Skill or capacity of the person to develop a certain physical or mental activity (operating, relating, designing processes, taking a calculated risk, using feedback from teachers, taking responsibility, setting personal goals, etc.).

Results - personal and/or professional performance.

- In an achievement motivation competence, the result is associated with continuous improvement in terms of hours of study/work dedicated, readings and complementary activities carried out, publication of papers or acknowledgements.

- In the case of innovation, it could be related to creative ideas, problem solving, new critical positions in certain situations or unique ways of studying.

In both cases the competencies linked to personality characteristics (motivation, traits, self-concept) are less visible 
while knowledge and behaviour are more identifiable. The formula incorporates modifications, namely:

\section{COMPETENCE $=$ INTENTION \\ (personality + motivation + self-concept + knowledge $)+$ ACTION + RESULT}

Some authors who have synthesized the definitions of competencies (Pérez Escoda and Sabariego Puig, 2015) consider the following characteristics to be the most relevant

- The concept is applicable to individuals or groups.

- It implies having knowledge, ability to know how to do and attitudes and behaviour to want to do or know how to be, all three interrelated.

- It circumscribes formal and informal capacities.

- Understanding them requires a combination of development, continuous learning and experience.

- Its potential is linked to the ability to take action.

- It incorporates contextual references of effectiveness and requires efforts to transfer them.

In this context we agree with Gonzci and Athanasou (1996) that competence-based education and training constitutes a coherent framework for learning and skill development. In any case, the important thing is to develop them in an appropriate way or, otherwise, not only will these competencies not be enhanced, but the structure of development of other skills will be damaged (Pavié, 2009).

Based on these arguments, it should be noted that competences have brought about a change of approach in the way learning, teaching and training are understood in all areas and for all people, since their orientation will be decisive in the degree of exclusion and inclusion of people in the future. To

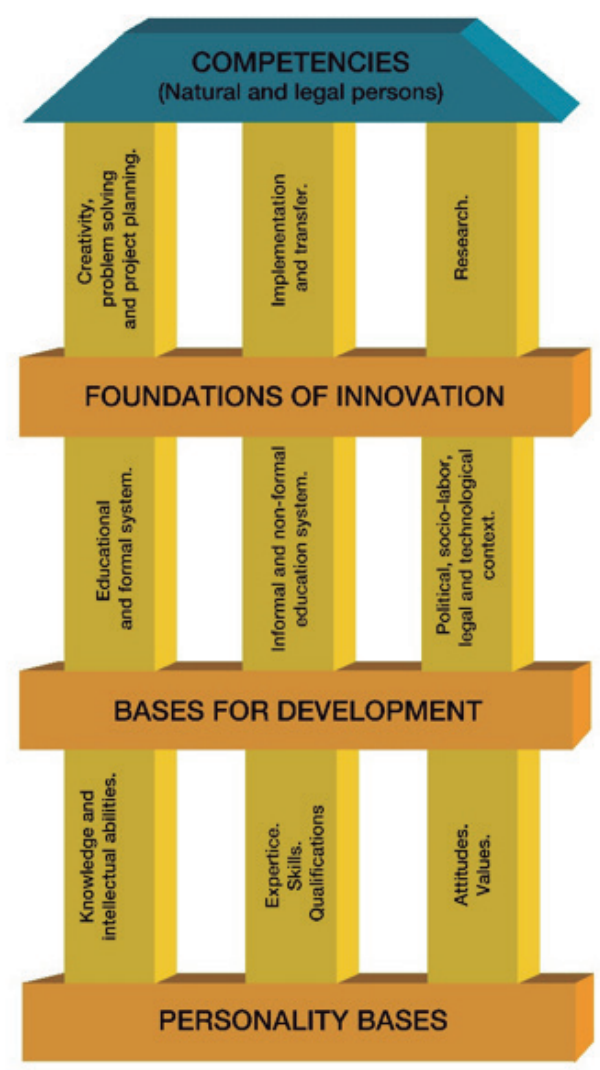

Chart 2. Basis for development of competencies tackle these problems many countries are choosing to increase the proportion of graduates in STEM (Science, Technology, Engineering and Mathematics) given the high employment rates associated with these fields due to their strong technological component but are finding that they are more exposed to global competition that is no longer led from Europe because some Asian countries are already ahead of their graduates in these degrees. The promotion of policies to encourage women in this context is another alternative, as is the promotion of these groups in green jobs related to science and technology.

In this context, digital talent is highly valued, but other social processes also emerge that generate induced labour demands where the human component takes on a new prominence in the form of face-to-face services. We refer to the effects of the differential ageing of the population on the planet or the incorporation of women into the labour market, since these facts have generated new demands for attention and care from other population groups, in the case of childcare, the elderly or dependents.

It will be important, therefore, to develop policies for the social dignification of these emerging sectors given their importance for economic development, social development and the well-being of people. Therefore, it seems logical to eliminate the high rate of prevalence of informal employment through the incorporation of professional qualification standards that lead us once again to value skills (CES Report, 2018).

It is important, therefore, to devote greater attention to reviewing how skills are being managed in our education systems when the emphasis continues to be on the internalisation of knowledge that expires and ceases to be useful or disappears in the short term. On the other hand, training in problem solving or coping with similar situations in different scenarios or carrying out analyses with multiple documentary sources or assuming different points of view in collaborative work environments are not extinguished because they adhere to people by integrating them into their personality.

To be able to, is to have grown, to be able to, is to face reality in a different way, to be able to, is to generate creative ideas to solve problems, to be able to... this approach makes it necessary to interpret formation as a process of shared growth and not as a process of individual accumulation. Approaching competencies from this perspective involves taking into account the bases of personality, together with the possibilities of development and orienting them toward the most humane competencies linked to innovation. This implies a revolution in learning and teaching processes, revitalizes multi-disciplinary training ecosystems as a viable and sustainable alternative, and supports cultural change in individuals and companies.

\section{Reflections and recommendations as a final synthesis}

Europe is facing a moment of transformation that is unprecedented, the cyclical socio-economic crises highlight the political, structural and cultural weaknesses in the EU to cope with deep-seated cultural changes. The regulatory framework has long contemplated the values and ideals needed to develop processes of change under new approaches (Europe 2020, Agenda 2030), but these intentions are not translated into practice as their promoters expect and, in many cases, they become exclusive because they are competitive. For this reason, we are going to recall some lessons learned from experience (Fullan, 1990, 1991, 1993, 1998, 2001; Escudero, 1999; Alvarez Arregui, 2017, 2018, 2019) that may be useful for moving into the future from other points of reference, in the case of universities: 
- Must adopt an eco-systemic perspective, involve people in the construction of the strategy (strategizing) and take into account the contributions of authors who have already explored these fields (Doyle, 1977; Bronfenbrenner, 1987; Tikunoff, 1979; Bunge, 1980; Bertalanffly, 1982; Gimeno Sacristán, 1998; De la Torre, 1998; Álvarez-Arregui, 2002, 2017, 2018; and Álvarez-Arregui and Rodríguez-Martín, 2014, 2015).

- Must value governance as a fundamental reference, so that institutional management must recognize that the information and knowledge they generate moves in complexity, hence people who perform tasks of direction, leadership and coordination must be able to relate, contextualize and globalize in a sequential and simultaneous way at different levels, which requires systemic learning.

- Must know that each institution is in itself an innovation ecosystem where any person and team can become an agent of change.

- Must foresee that, when the educational agents in charge of developing the strategies do not visualize that they are participating in it, they will be the ones who will demand the changes in management, in the participation systems, in the search for the necessary funding, in the hiring models or in the methodologies to be used.

- Must remind rectors, deans, teachers, and administration and service teams that the functions defined in the legislative framework clearly specify that the development of their institutions, of competing professionals, of their communities, and of users depends to a great extent on how their visions, policies, and strategies are deployed.

- Must be aware that the capacity of influence of people who exercise leadership is associated with the use of resources (money, information, materials, space, time, etc.)); their position in the organization charts; their charisma (physical, relational or communicative traits); their values (credibility, what they say and what they do); their training (background in management, teaching and research); the opportunity to intervene (based on the support obtained in their access to positions); their leadership style (individual consideration, intellectual stimulation, delegation of leadership, institutional projection and community orientation) and their ethics (improving the quality of life of the people involved).

- Must promote transparent and formative monitoring and evaluation models to generate confidence in the promoters and the processes, since this approach will favour communication, the extension of leadership, coordination and the construction of a joint vision that will make it possible to lay the foundations of an organisational culture favourable to continuous learning, change, improvement and inclusion.

- Must devote some time and effort to the integration of the different subcultures, since the risk of irretrievable breakdowns among them will be reduced and people will participate in the vision and institutional strategies.

- Must communicate the vision at different times to sensitize the community and they must do so situationally top-down, bottom-up, horizontally and transversally if they are to join forces and strengthen initiatives for change.

- Must addressed administrative, curricular and research projects with a transdisciplinary, eco-educational and intercultural perspective.
- Must remember that change is a permanent journey, that you cannot command what must be done, that problems are a source of learning, that the vision is built among all people, that individualism and collectivism have equal power, that neither centralization nor decentralization works in isolation, that anyone can become an agent of change, that internal organizational cultures sift through external prescriptions, that internal and/or external relationships can be critical, that organizational knowledge management is complex, that mimetic adaptations of external guidelines are standardized, do not crystallize and become bureaucratic; and that the situation gets worse when there is a high disaffection of people with the projects and/or when users do not identify with external or institutional proposals since these and other issues end up translating into a loss of trust and credibility in the programmes, institutions and the system.

Experience also tells us that, in the face of a complex and multidimensional reality, simple, exclusive and standardised solutions are no longer viable. In our case we advocate the development of unique projects that incorporate in their design, implementation and impact analysis the different agents of the political, legal, economic and academic system. In this scenario, we share those initiatives that deepen in structural pluralism from interpretative, critical and complex approaches, overcoming the limitations derived from bureaucratic, competitive and standardized models, to integrate the best of the public, private and voluntary sectors through an eco-educational, systemic, transdisciplinary, entrepreneurial, ethical, transparent and inclusive view.

\section{Referencias / Referents}

Álvarez-Arregui, E.

(2002). Acción directiva y cultura escolar. Influencia del liderazgo en el desarrollo institucional de los centros educativos. Oviedo: Universidad de Oviedo.

(2010a). La Universidad desde una perspectiva de cambio: En busca de la excelencia. I Congresso Ibero-Brasileiro nos días 29 y 30 de abril en Elvas e 1 e 2 de Maio de 2010 en Mérida e Cáceres.

(2010b). La universidad ante la excelencia: Posibilidades y límites en períodos de incertidumbre. XI Congreso Internacional de Instituciones Educativas. Cuenca: Universidad de Castilla-La Mancha.

(Colaborador) (2017a). Formación Curricular en Diseño para Todas las Personas en Educación. CRUE. Universidades Españolas.

(Coord.) (2017b). Universidad, Investigación y Conocimiento: Avances y Retos. Oviedo: Servicio de Publicaciones de la Universidad de Oviedo.

(Coord.) (2018). Universidad, Investigación y Conocimiento: comprensión e intervención en una sociedad compleja. Oviedo: Servicio de Publicaciones de la Universidad de Oviedo.

(Coord.) (2019). Universidad, Investigación y Conocimiento: La Transversalidad como referente del desarrollo profesional y personal. Oviedo: Servicio de Publicaciones de la Universidad de Oviedo.

Álvarez-Arregui, E. y Rodríguez-Martín, A.

(2015a). Aprender a mirar las organizaciones desde una visión inclusiva. Avanzando desde la práctica hacia un modelo ecosistémico de formación y gestión. Oviedo: Editorial Ediuno.

(2015b). Inspirando el cambio en Educación. Ecosistemas de formación para aprender a emprender en la Universidad de 
Oviedo. Estudios Pedagógicos. Universidad Austral de Chile, vol. 41, 9-29.

Álvarez-Arregui, E.; Rodríguez-Martín, A.; Madrigal Maldonado, R.; Grossi-Sampedro, B. G.; y Arreguit, X. (2017). Ecosystems of media training and competence. International assessment of its implementation in Higher Education. Comunicar, 51, XXV, 105-114.

Álvarez-Arregui, E.; Rodríguez-Martín, A.; San Fabián Maroto, J. L.; y Álvarez Fernández, M.ํㅡ. V. (2011). Una nueva encrucijada para las Políticas Públicas en Educación Superior. La universidad como inversión o la universidad como inversora. XXV Simposio Brasileiro de Política e Administraçao da Educaçao. Sao Paulo-Brasil: FEAE.

Álvarez-Arregui, E.; Rodríguez-Martín, A.; y Ribeiro Gonçalves, F. (2013). Los ecosistemas de formación blended learning en la práctica universitaria. Valoración de los estudiantes sobre su implementación. Revista Portuguesa de Educaçao, 26 (1), 143177.

Bertalanffy, L. (1982). Teoría general de sistemas. Madrid. FCE.

Bricall, J. M. (2000). Universidad 2mil. Madrid: CRUE.

Bronfenbrenner, V. (1987). Ecología del desarrollo Humano. Barcelona. Paidos.

Bunge, M. (1989). La investigación científica. Barcelona: Ariel.

Castells, M.

(1999). La era de la información. Fin de milenio. Vol. 3. Madrid: Alianza.

(2000a). La era de la información. El poder de la identidad. Vol. 2. Madrid: Alianza.

(2000b). La era de la información. La sociedad red. Vol. 1. Madrid: Alianza.

Comisión de Comunidades Europeas (2003). El papel de las universidades en la Europa del Conocimiento. COM, 58 final. Bruselas.

De la Torre, S. (Coord) (1998). Cómo innovar en los centros educativos. Estudio de casos. Madrid. Editorial Escuela Española.

De Miguel Díaz, M. (Dir.) (2005). Modalidades de enseñanza centradas en el desarrollo de competencias. Orientaciones para promover el cambio metodológico en el Espacio Europeo de Educación Superior. Oviedo. Universidad de Oviedo/MEC.

Dosi, G., Freeman, C., Richard, N., Silverberg, G. and Soete, L. (Eds.) (1990). Technical Change and Economic Theory. Londres: Pinter.

Doyle, W. (1986). Context representation in teacher's definitions of academic work. Journal of Curriculum Studies, 18 (4), 365379.

Escudero, J. M. (1999). El cambio en educación, las reformas y la renovación pedagógica. ESCUDERO, J. M. (ed.). Diseño, desarrollo e innovación. Currículum. Madrid. Síntesis. 76-96.

Escudero, R. y Martínez, B. (2012). Las políticas de lucha contra el fracaso escolar: ¿programas especiales o cambios profundos del sistema y la educación? Revista de Educación, número extraordinario, 174-193.

Etzkowitz, H.; Leydesdorff, L. (1997). University and the Global Knowledge Economy. A triple Helix of University - Industry Government Relations. London: Pinter Publishers.

Fisher, R.; Ury, W. y Patton, B. (2002). Obtenga el sí. El Arte de Negociar Sin Ceder. Barcelona: Gestión 2000.

Fullan, M.

(1990). El desarrollo y la gestión del cambio. Revista de Innovación e Investigación Educativas, 5, 9-22.

(1991). The new meaning of Educational change. Nueva York/
Londres. Teachers College. Press/Cassell. (Traducida al castellano por Octaedro-Barcelona en 2002 bajo el título Los nuevos significados del cambio en educación).

(1993). Change forces. The depths of educational reform. Londres. The Falmer Press.

(1998). The Mening of Educational Change. A Quarter of a Century of Learning. HARGREAVES, A. y OTROS (Eds.). Internacional Handbook of Educational Change. Kluwer Academic Publising.

(2001). Emoción y esperanza: conceptos constructivos para tiempos complejos. HARGREAVES, A. (Coord.). Replantear el cambio educativo. Un enfoque renovador Madrid. Amorrortu. 296-317.

Gimeno Sacristán, J.

(1998). Poderes Inestables en educación. Madrid. Morata.

(2001). Educar y convivir en la cultura global. Madrid: Morata.

González, J. y Wagenaar, R. (2003) Tuning Educational Structures in Europe. Informe final, Fase uno. Documento policopiado.

Gonzci, A. y Athanasou, J. (1996). Instrumentación de la educación basada en competencias. Perspectivas de la teoría y práctica en Australia, en MERTENS, L. (1996). Competencia laboral: sistemas, surgimientos y modelos. CINTERFOR/OIT. Montevideo, Uruguay. http://cinterfor.org.uy

Le Goff, J. (1993). Los intelectuales en la Edad Media. Madrid: Gedisa.

López Gómez, E. (2015). La tutoría en el EESS: propuesta, validación $y$ valoración de un modelo integral. Tesis doctoral inédita.

Morín, E.

(1995). Introducción al Pensamiento Complejo. Barcelona. Gedisa.

(1998). Sobre la reforma de la universidad. PORTA, J. \& LLADONOSA, M. (Coords.). La universidad en el cambio de siglo. Madrid. Alianza/Fundación 700 Aniversario de la Universidad de Lleida. 19-28.

OCDE (1999). University research in transition. París: OCDE.

Pavié, A. (2009). Formación docente y enfoque por competencias, en Desarrollo e Integración de la Educación Superior. CASTRO, E. y RODRÍGUEZ ROJO, M. (Eds). ISBN 978-956-332-121-0. Edición electrónica. Osorno: Ediciones Red Urel/Urelva, pp. 20- 41.

Pérez-Escoda, N. y Sabariego Puig, M. (2015). Valoración de la efectividad de la formación en competencias emocionales en educación superior. AIDIPE (Ed.), Investigar con y para la sociedad (Vol.2, pp.965-976). Cádiz, España: Bubok.

Perrenoud, $P$.

(2004a). Desarrollar la práctica reflexiva en el oficio de enseñar. Barcelona. Graó.

(2004b). Diez nuevas competencias para enseñar. Barcelona. Graó.

Ruiz Corbella, M. (2006). La universidad y el mercado del aprendizaje. Claves para comprender el concepto de competencia. 93-114. MURGA MENOYO, Ma․ \& QUICIOS GARCÍA, Mª . P. (coord.). La reforma de la universidad. Cambios exigidos para la nueva Europa. Madrid. Dykinson.

Saura, G. y Bolivar, A. (2019). Sujeto Académico Neoliberal: Cuantificado, Digitalizado y Bibliometrizado. Revista Iberoamericana sobre Calidad, Eficacia y Cambio en Educación, $17(4), 9-26$

Senge, P. M. (1990). The Fifth Discipline. The Art and Practice of the Learning Organization. N. Y. Dobuleday.

Tikunoff, W. J. (1979): Context Variables of Teaching-Leaming Events, en BENNET, S. y MCNAMARA: Focus on Teaching. London: Longman. 
